\title{
BIO-DEVELOPMENT OF MOTORWAY NETWORKS IN THE NETHERLANDS: A SLIME MOULD APPROACH
}

\section{Final version of this paper is published in Advances in Complex Systems (2012) DOI: $10.1142 /$ S0219525912500348}

\author{
Andrew Adamatzky ${ }^{1}$,Michael Lees ${ }^{2}$ and Peter M.A. Sloot ${ }^{2,3,4}$ \\ 1 University of the West of England, Bristol BS16 1QY, United Kingdom \\ andrew . adamatzky@uwe.ac.uk \\ 2 Nanyang Technological University, Singapore \\ mhlees@ntu.edu.sg \\ 3 University of Amsterdam, Amsterdam, The Netherlands \\ 4 National Research University ITMO, Russia \\ p.m.a.sloot@uva.nl
}

\begin{abstract}
Plasmodium of acellular slime mould Physarum polycephalum is a very large eukaryotic microbe visible to the unaided eye. During its foraging behaviour the plasmodium spans sources of nutrients with a network of protoplasmic tubes. In this paper we attempt to address the following question: is slime mould capable of computing transport networks? By assuming the sources of nutrients are cities and protoplasmic tubes connecting the sources are motorways, how well does the plasmodium approximate existing motorway networks? We take the Netherlands as a case study for bio-development of motorways, while it has the most dense motorway network in Europe, current demand is rapidly approaching the upper limits of existing capacity. We represent twenty major cities with oat flakes, place plasmodium in Amsterdam and record how the plasmodium spreads between oat flakes via the protoplasmic tubes. First we analyse slime-mould-built and man-built transport networks in a framework of proximity graphs to investigate if the slime mould is capable of computing existing networks. We then go on to investigate if the slime mould is able calculate or adapt the network through imitating restructuring of the transport network as a response to potential localalized flooding of the Netherlands.
\end{abstract}

Keywords: bio-inspired computing, Physarum polycephalum, pattern formation, The Netherlands motorways, road planning 


\section{Introduction}

The approximation or computation of shortest path transportation networks has drawn significant attention from the field of Unconventional Computing Sciences. Nature-inspired computing paradigms and experimental implementations have been successfully applied to calculation of a minimal-distance path between two given points in a space or a road network. Computational models of ant-based optimisation have been shown to be an effective way of developing novel approaches towards load-balancing of telecommunications [12], which indeed involves dynamical design of transport links for packets. Other work includes a shortest-path problem solved in experimental reaction-diffusion chemical systems [1], gas-discharge analog systems [24], spatially extended crystallization systems [7, formation of fungi mycelian networks [15] and plasmodium of Physarum polycephalum [20].

Amongst all experimental prototypes of path-computing devices slime mould Physarum polycephalum is perhaps the most cost efficient biological substrate available, coupled with fact that it is both easy to cultivate and observe, it makes an excellent computational substrate. These are the main reasons we adopt it for this work.

Acellular slime mould Physarum polycephalum has quite a sophisticated life cycle [27], which includes various stages such as: fruit bodied, spores, single-cell amoebas, and syntsyncytium. Plasmodium is a vegetative stage of Physarum polycephalum, it is a syncytium, a single cell, where many nuclei share the same cytoplasm. The plasmodium consumes microscopic particles, and during its foraging behaviour the plasmodium spans scattered sources of nutrients with a network of protoplasmic tubes. The protoplasmic network is usually optmized to cover all sources of food while still managing to guarantee robust and quick distribution of nutrients in the plasmodium body. Plasmodium's foraging behaviour can be interpreted as computation, with data represented by spatial distribution of attractants and repellents, and results represented by the structure of protoplasmic networks [10]. Plasmodium is capable of solving computational problems with natural parallelism, namely shortest path [20] and hierarchies of planar proximity graphs [5], computation of plane tessellations [26], execution of basic logical computing schemes [29,9], and natural implementation of spatial logic and process algebra [25]. For further examples see the overview of Physarum-based computers in [10].

In previous work [4 we have evaluated the road-modeling potential of $P$. polycephalum, however, previous results were inconclusive. A step forward 
biological-approximation, or evaluation, of man-made road networks was done in our previous work on approximation of United Kingdom motorways and Mexican Federal highways by plasmodium of Physarum polycephalum [8,11]. In both cases it was shown that transportation links constructed by plasmodium sufficiently determines man-made motorways, with some pernicious differences. Comparing results for United Kingdom and Mexico we found that shape of a country and spatial configuration of urban areas or cities sufficiently determines behaviour of the plasmodium. More experiments are necessary to provide generalisation, in order to develop a theory of slime-mould based road planning and urban development.

In this paper we hope to move towards a more general understanding of slime moulds capability to compute road networks by investigating the roads in the Netherlands. The Netherlands presents an excellent case study as it has the highest density motorway network in Europe. Moreover, the demand on the system is at levels which are reaching current limits, with a total length of $132,397 \mathrm{~km}$ and usage of $140 \times 10^{9}$ people per $\mathrm{km}$ per yea ${ }^{1}$. Such highoccupancy may pose a need for urgent expansion of the transport networks and a better understanding of the limitations to that growth. The Netherlands is also at risk of significant flooding 2 .

The remainder of this paper is structured as follows. We delinate the experimental method and setup in section 2. Section 3 presents the principal experimental results, which are then analysed in a framework of proximity graphs in section 4. Restructuring of Physarum-approximated transport links for the case of partial flooding of the Netherlands is described in section 5 . The paper then concludes with a summary of the work and ideas for further studies in section 6 .

\section{Methods}

All experiements are conducted with Plasmodium of $P$. polycephalum that is cultivated in a plastic container. The Plasmodium are first placed on paper kitchen towels, sprinkled with still water and fed with oat flakes 3 . The experiments are conducted in $120 \times 120 \mathrm{~mm}$ polyestyrene square Petri dishes with rounded corners. The Plasmodium will eventually grow on Agar plates, which are cut into the shape of the Netherlands. The Agar plates are formed using $2 \%$ agar gel (Select agar, Sigma Aldrich).

\footnotetext{
1 www.autosnelwegen.nl

2 http://urbanflood.eu/default.aspx

3 Asda's Smart Price Porridge Oats
} 


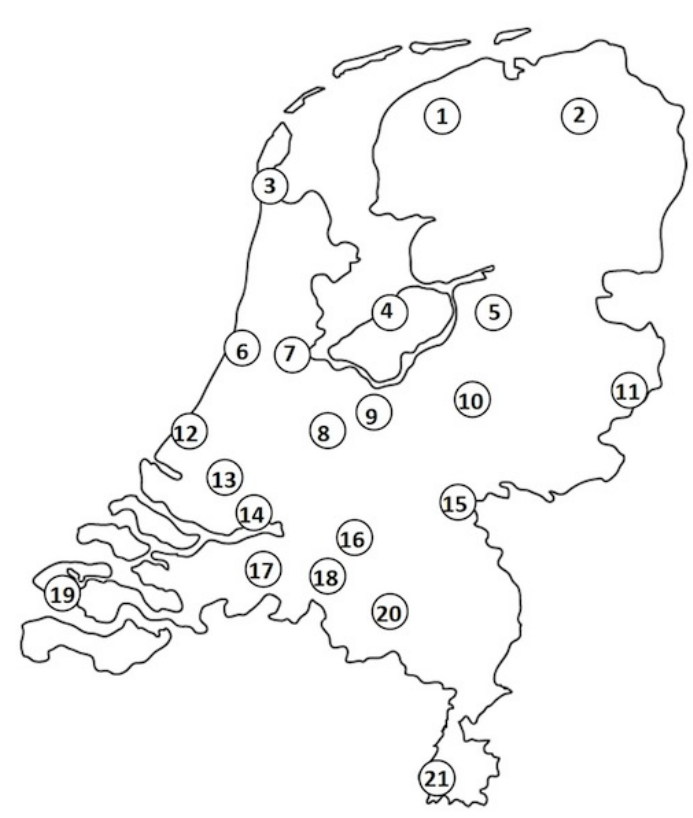

(a)

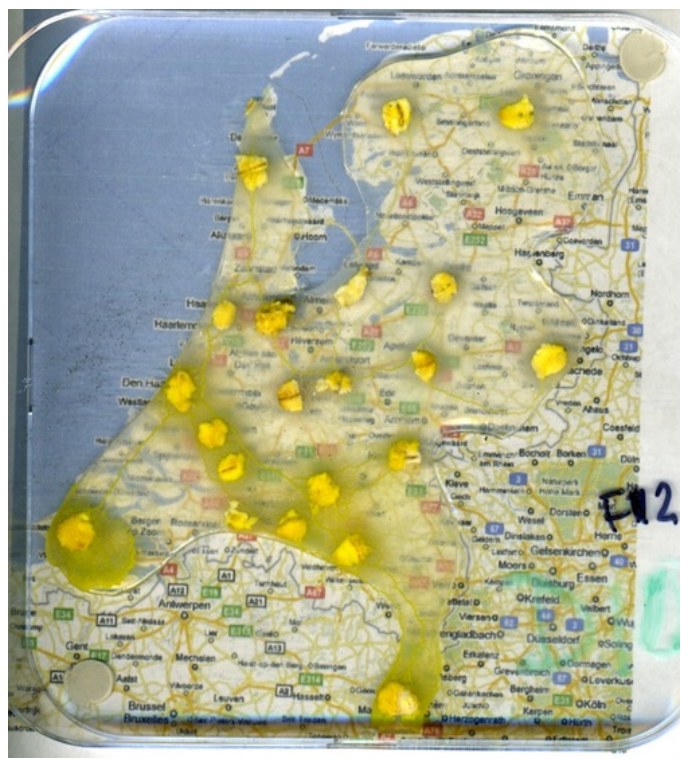

(c)

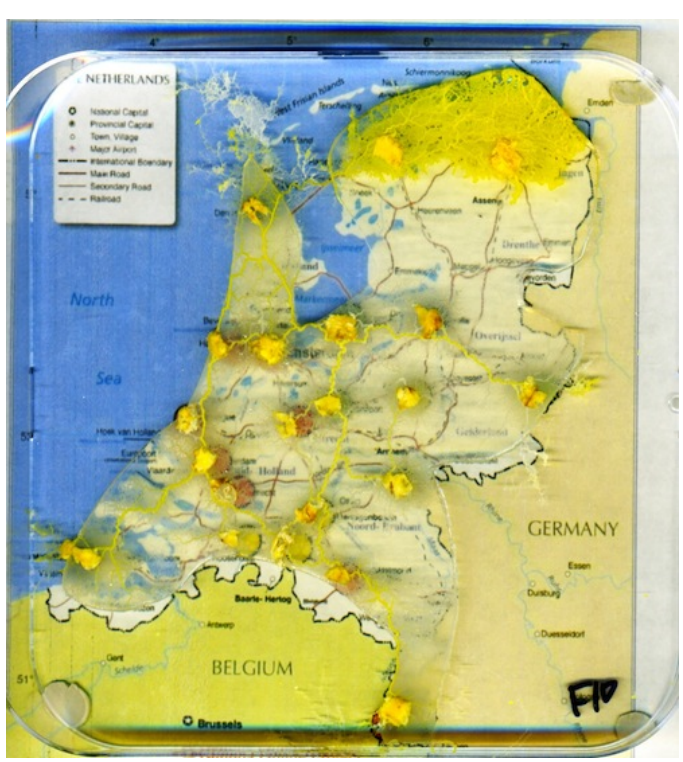

(b)

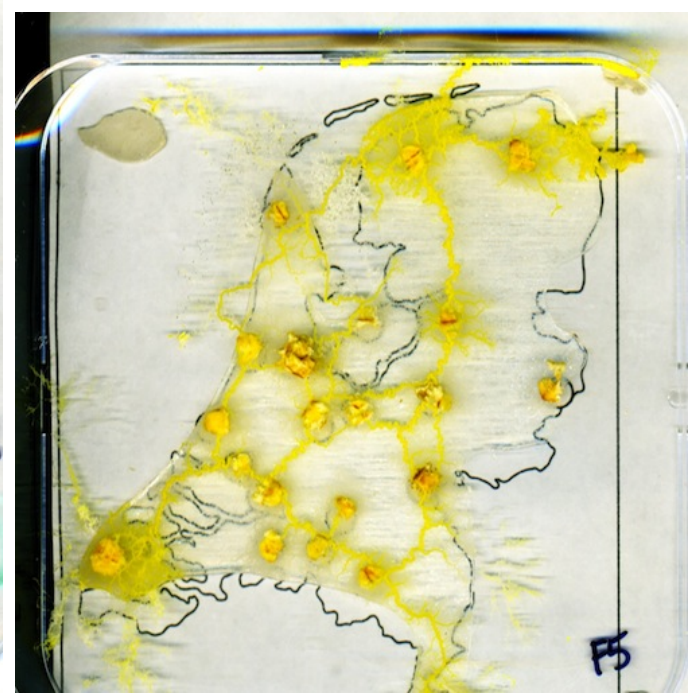

(d)

Fig. 1. Experimental basics. (a) Outline map of the Netherlands with twenty one sources of nutrients indicated. (b)-(d) Snapshots typical setups: urban areas are represented by oat flakes, plasmodium is inoculated in Amsterdam, the plasmodium spans oat flakes by protoplasmic transport network.

All experiments consider the twenty one most populous urban areas in the Netherlands (Fig. 17):
(1) Leeuwarden
(3) Den Helder
(2) Groningen
(4) Lelystad 
(5) Zwolle

(6) Haarlem

(7) Amsterdam

(8) Utrecht

(9) Amersfoort

(10) Apeldoon

(11) Enschede

(12) Den Haag

(13) Rotterdam
(14) Dordrecht

(15) Nijmegen

(16) Hertogenbosch

(17) Breda

(18) Tilburg

(19) Middelburg

(20) Eindhoven

(21) Maastricht.

Further we refer to the urban regions as $\mathbf{U}$. The regions in $\mathbf{U}$ are projected onto the gel in the following manner: oat flakes are placed in the positions of each region (Fig. 1b). At the beginning of each experiment a piece of plasmodium, usually already attached to an oat flake, is placed in Amsterdam (region 7 in Fig. 11).

The Petri dishes with plasmodium are kept in darkness, at a temperature of between 22 and $25 \mathrm{C}^{\circ}$, except for short periods of observation and image recording. Periodically the dishes are scanned using an Epson Perfection 4490 scanner. Scanned images of dishes are enhanced to increase readability of the image, this is done by increasing saturation and contrast (saturation is increased to 55 and contrast to 40). A total of 62 experiments were conducted.

To ease understading of experimental images we provide complementary binary version of each image, where appropriate. In these images each pixel of the color image is assigned a black color if red $R$ and green $G$ components of its RGB color exceed some specified thresholds, $R>\theta_{R}, G>\theta_{G}$ and the blue component $B$ does not exceed some threshold value $B<\theta_{B}$; otherwise, the pixel is assigned a white color (exact values of the thresholds are indicated in the figure captions as $\left.\Theta=\left(\theta_{R}, \theta_{G}, \theta_{B}\right)\right)$.

\section{Transport links via foraging}

In the following we present experimental results which show the the plasmodium is capable of computing, or calculating, the transport links between each of the twenty one most populus areas of the Netherlands.

In a laboratory experiment, illustrated in Fig. 2, the following chain of events unfolds (dynamics of colonisation is schematically represented in Fig. 3). An oat flake colonised by plasmodium was placed on top of the oat flake representing Amsterdam. In 12 hours the plasmodium follows gradients of chemoattractants, links Amsterdam with Haarlem, and propagates towards Utrecht and 


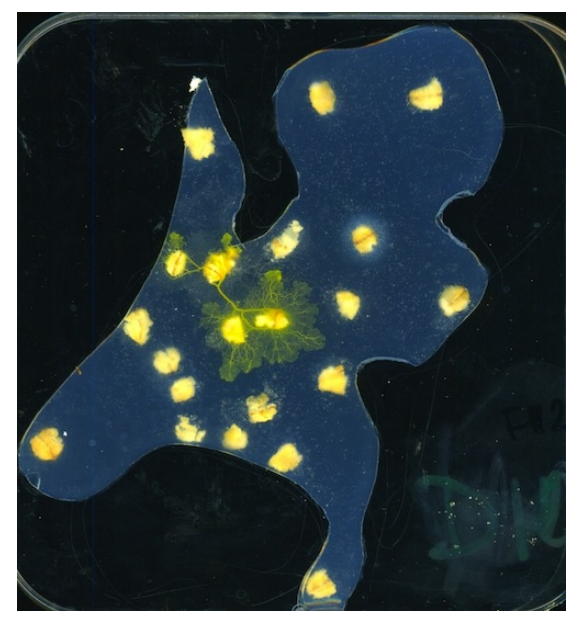

(a) $t=12 \mathrm{~h}$

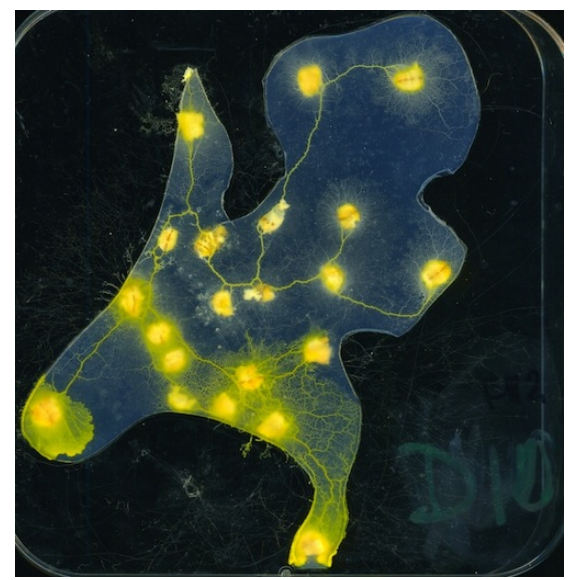

(c) $t=57 \mathrm{~h}$

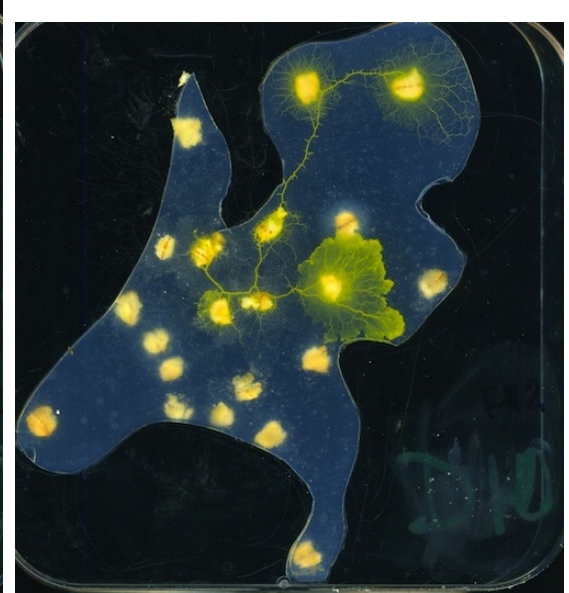

(b) $t=34 \mathrm{~h}$

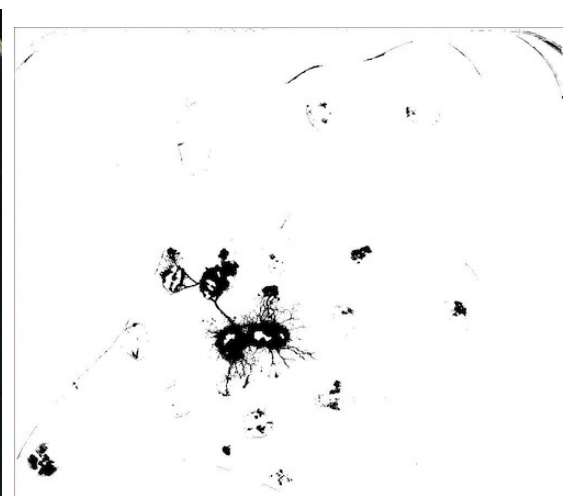

(d) $t=12 \mathrm{~h}$

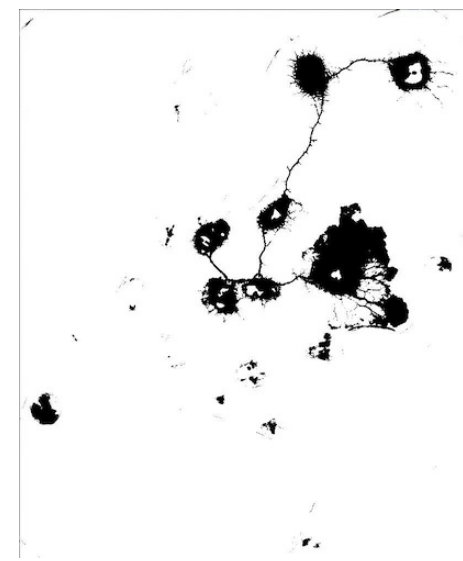

(e) $t=34 \mathrm{~h}$

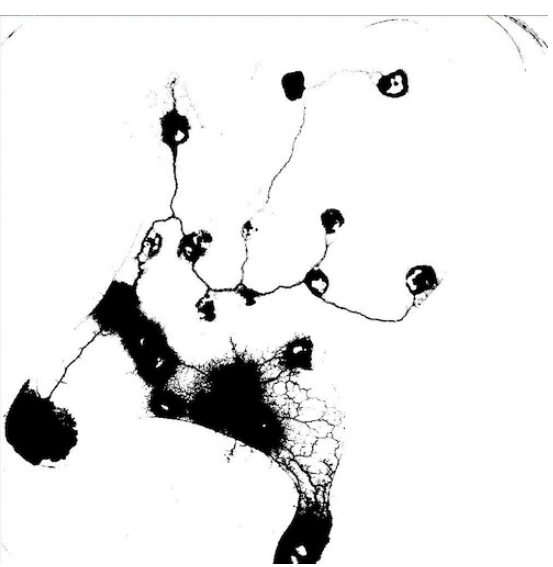

(f) $t=57 \mathrm{~h}$

Fig. 2. Illustrative example of plasmodium development on configuration of cities represented by oat flakes: (a)-(c) scanned image of experimental Petri dish. Time elapsed from inoculation is shown in the sub-figure captions. (d)-(f) binary images, $\Theta=(100,100,100)$. 


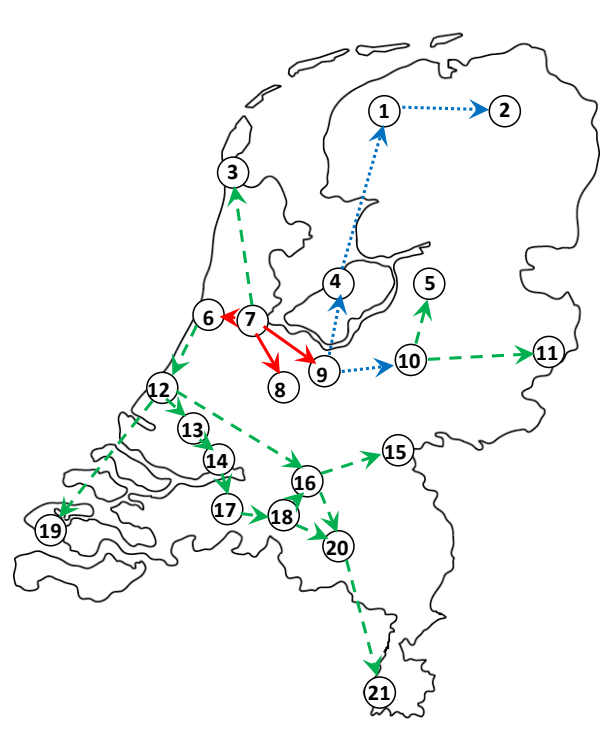

(a)

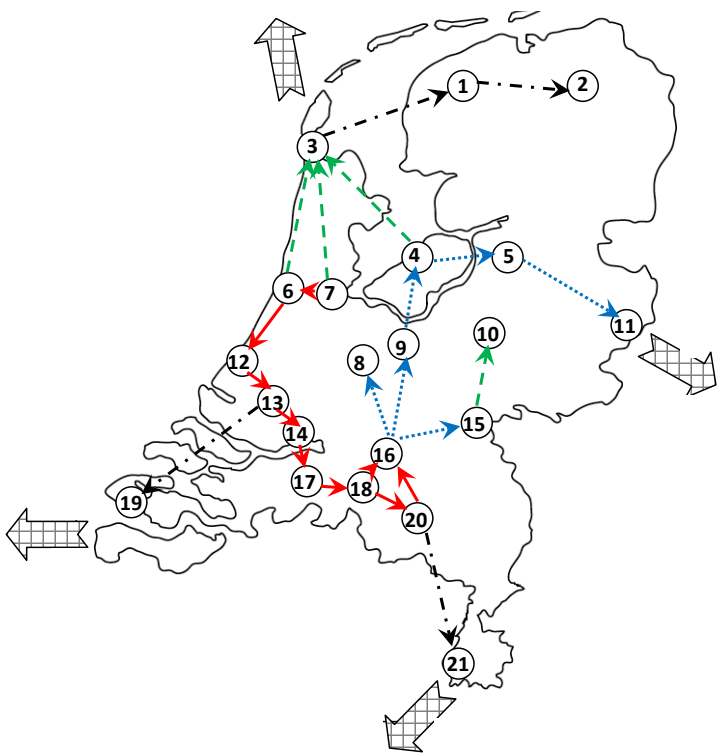

(b)

Fig. 3. Diagram of colonisation dynamics derived from experiments Fig. 2 (a) and Fig. 4 (b): links developed in 12 hours after inoculation are shown by red solid arrows, in 34 hours by blue dotted arrows, in 57 hours by green dashed lines, in 80 hours by dash-dotted lines. Large mesh-patterned arrows indicate migration of plasmodium outside the country.

Amersfoort, spreading in all directions except north-west (Fig. 2ad). After 34 hours the plasmodium colonizes Leeuwarden and Groningen. It develops clearly visible protoplasmic tubes, which represent a transport link Amersfoort - Lelystad - Leeuwarden - Groningen (Fig. 2be). In the same time interval the plasmodium colonises Apeldoon and start propagations towards Zwolle and Enschede (Fig. 2be).

After a total of 57 hours the plasmodium connects Apeldoon with Enschede and Zwolle by protoplasmic tubes and colonised south-west part of the country. Namelym, the plasmodium links Haarlem and The Hague and builds a route from The Hague to Middelburg and a link Hague - Rotterdam - Dordrecht - Breda - Tilburg - Hertogenbosch (Fig. 2cf, Fig. 3a, green dashed lines). At the same time the plasmodium forms a protoplasmic tube directly connecting Amsterdam Den Helder, and The Hague with Hertogenbosch, and develops the links Hertogenbosch - Nijmegen and Tilburg - Hertogenbosch - Eindhoven - Maastricht (Fig. 20f).

We observe that the dynamics of colonisation is non-uniform (Fig. 3a). The Plasmodium does not spread or diffuse in all directions simultaneously but rather colonises north-north-west part of the country first and only then explores south-south-west. This may be due to the fact that centres of activity (biochemical oscillators) form during propagation, and the contractive waves 


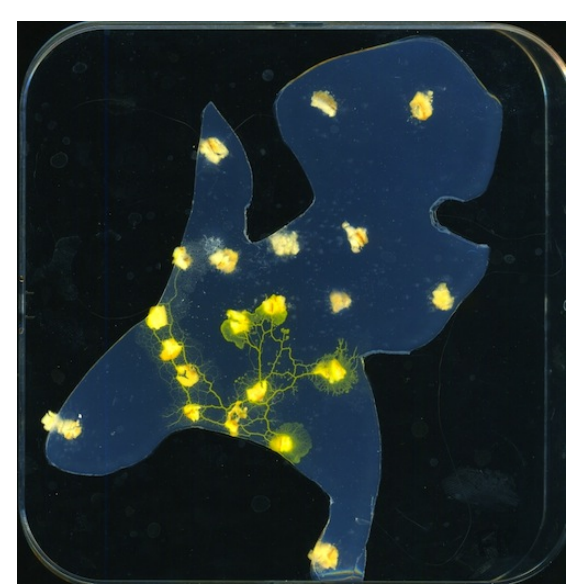

(a) $t=12 \mathrm{~h}$

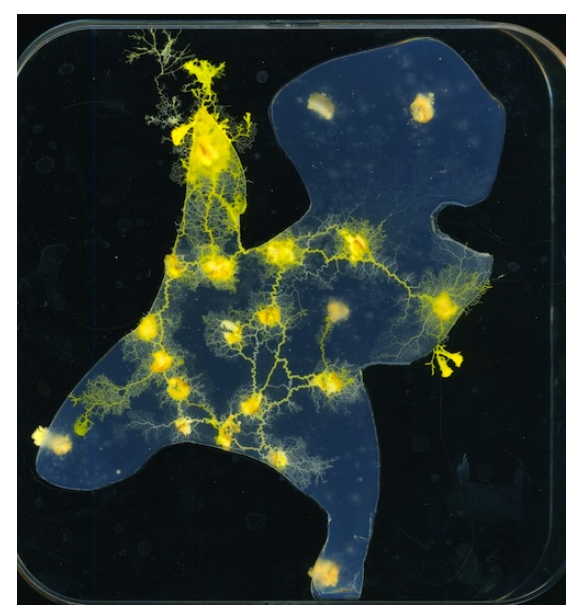

(c) $t=57 \mathrm{~h}$

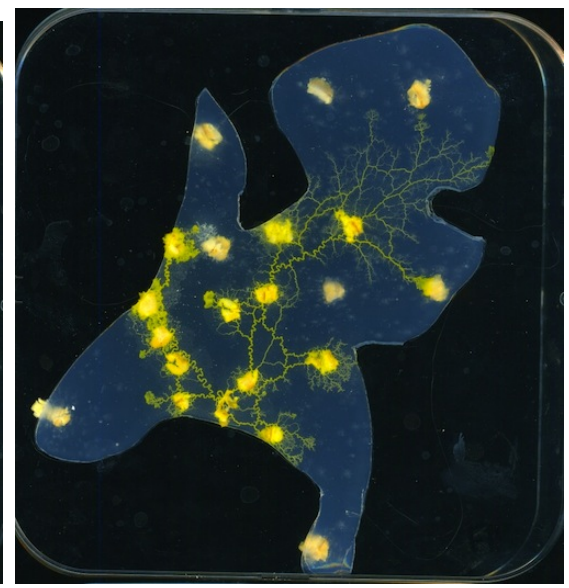

(b) $t=34 \mathrm{~h}$

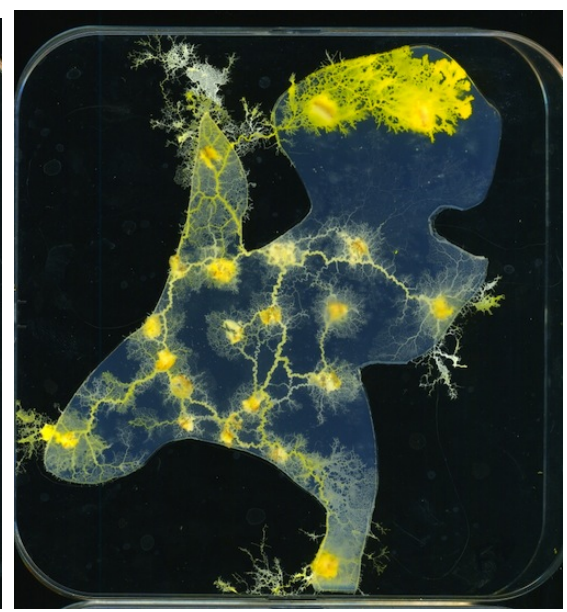

(d) $t=80 \mathrm{~h}$

Fig. 4. Plasmodium spreads beyond 'dedicated' experimental domain: (a)-(d) scanned image of experimental Petri dish. Time elapsed from inoculation is shown in the sub-figure captions. (e)-(h) binary images, $\Theta=(100,100,100)$.

evoked by the oscillators that force the protoplasm to move towards the oscillators. Therefore, if an oscillator is formed in the north part of plasmodium, then propagation in all other directions would be suppressed.

The plasmodium of Physarum polycephalum rarely repeats itself in experimental trials. The overall or average pattern, as we will discuss further in the paper, may be the same but a myriad of variations are possible in the course of plasmodium's spatial development.

Outperforming (spreading out of the dedicated area) and under-performing (not colonising the whole area) are typical examples of the varieties in plasmodium behaviour. These two examples are illustrated and discussed below.

In a substantial number of laboratory experiments, the plasmodium did not 


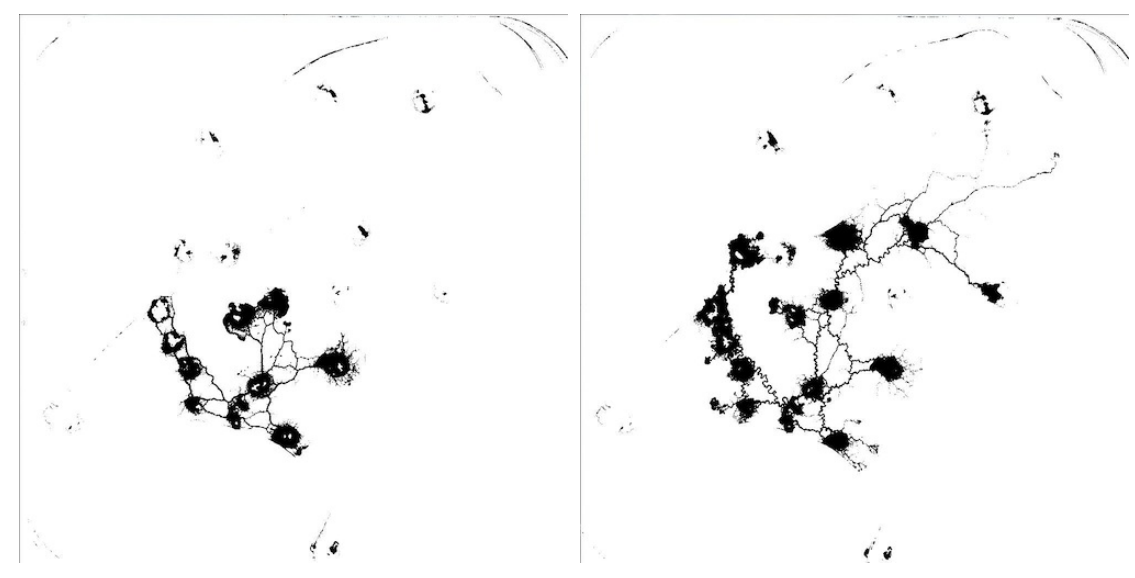

(e) $t=12 \mathrm{~h}$

(f) $t=34 \mathrm{~h}$

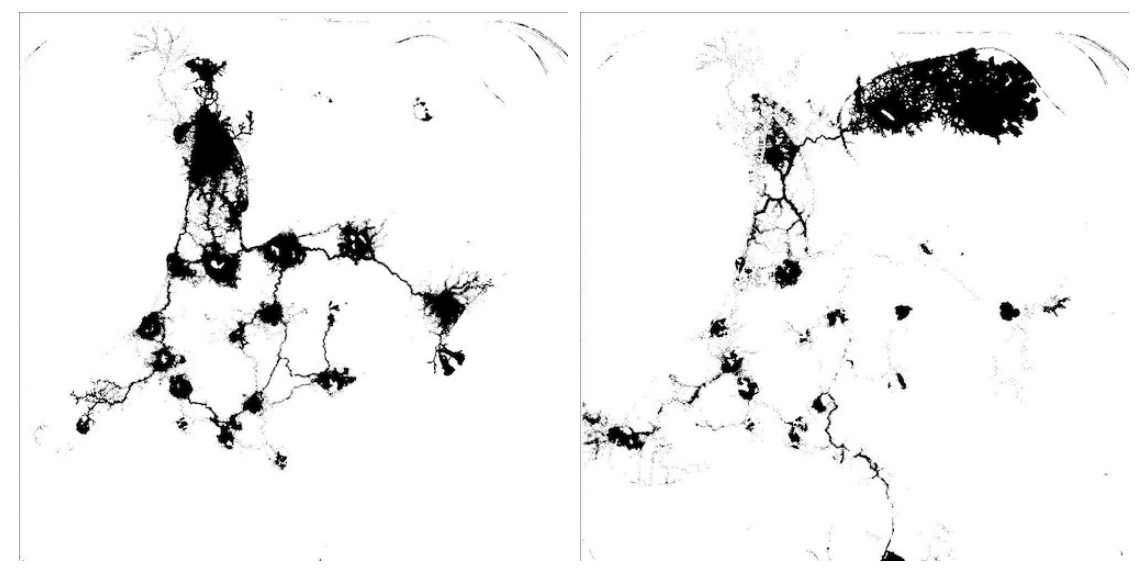

(g) $t=57 \mathrm{~h}$

(h) $t=80 \mathrm{~h}$

Fig. 4. Continued.

stop its foraging activity even when all sources of nutrients were occupied and the whole agar plate was explored. As shown in Fig. 4 a vigorous plasmodium can spread over surrounding Petri dishes, trying to settle on bare plastic.

In this experiments Plasmodium starts its colonisation in Amsterdam as before. It's colinization is more aggresive in this case and it colonises Haarlem, Den Haag, Rotterdam, Dordrecht, Breda, Tilburg, Hertogenbosch, Eindhoven within the first twelve hours. A pronounced protoplasm transport link is established connecting these cities in a chain (first 12 hours from the moment of inoculation, Fig. 4ae). 34 hours after inoculation the plasmodium sprawls from Hertogenbosch to Utrecht, Amersfoort and Apeldoon, and then builds a transport link Amersfoort-Lelystad-Zwolle-Enschede (Fig. 4 bf).

Protoplasmic tubes connecting Haarlem, Amsterdam, Lelystad with Den Helder are grown simultaneously after 57 hours of the experiment. By the same time plasmodium also connects Nijmegen with Apeldoon (Fig. 4 $\mathrm{cg}$ ). Protoplasmic transport links Den Helder - Leeuwarden - Groningen, Rotterdam - Middel- 
burg and Eindhoven - Maastricht are developed by the 80th hour of plasmodium's foraging activity (Fig. 4 dh). A Schematic illustration of the colonisation dynamics is shown in Fig. $3 \mathrm{~b}$.

Plasmodium starts to show overperformance after 57 hours of the experiment. It sprawls from Den Helder north-westward and from Enschede south-eastward onto bare plastic of the experimental container (Fig. $4 \mathrm{~kg}$ ). The plasmodium does not propagate on the plastic long enough and retracts in few hours (this can be seen in Fig. 4dh). Another sprawling takes place by the 80th hour of experimentation, when plasmodium propagates westward of Middelburg and sout-eastward of Maasrticht (Fig. 4 $\mathrm{dh}$ ). See also diagrams of sprawling outside the county in Fig. 3b. Also notice how the plasmodium dynamically changes its foraging strategy (Fig. 4). It first attempts to colonise cities in north-east part of the country but then abandons the attempt and move to north-east later via the IJsselmeer lake.

In some experiments the plasmodium never manages to span all cities, and fails to collanize some oat flakes. An example is shown in (Fig. 5), 65 hours after inoculation the plasmodium colonises the majority of the Netherlands and establishes a network of protoplasmic tubes over most of the cities represented by oat flakes (Fig. 5 $\mathrm{cf}$ ). Later it goes into a hibernation stage and forms a sclerotium. However, at no moment of its development does the plasmodium even approach Middelburg. Such situations are rather atypical and did not happen often in our experiments.

As illustrated above, plasmodium is quite an unpredictable creature and the patterns formed by its protoplasmic networks in any two experiments rarely match each other exactly. Thus we generalise results of our experiments by constructing a probabilistic Physarum graph. A Physarum graph is a tuple $\mathbf{P}=\langle\mathbf{U}, \mathbf{E}, w\rangle$, where $\mathbf{U}$ is a set of 21 cities, $\mathbf{E}$ is a set edges, and $w: \mathbf{E} \rightarrow[0,1]$ is a probability-weights of edges from $\mathbf{E}$. For every two cities $a$ and $b$ from $\mathbf{U}$ there is an edge connected $a$ and $b$ if a plasmodium's protoplasmic link is recorded at least in one of $k$ experiments, and the edge $(a b)$ has a probability calculated as a ratio of experiments where protoplasmic link (ab) occurred to the total number of experiments $k$. We do not take into account exact configuration of the protoplasmic tubes but merely their existence, e.g. protoplasmic tubes linking Eindhoven with Maastricht always positioned inside the Netherland territory but corresponding edge in Physarum graph represents the tubes by straight line. We also consider threshold $\theta \in[0,1]$ Physarum graphs $\mathbf{P}(\theta)$, defined as follows: for $a, b \in \mathbf{U},(a b) \in \mathbf{E}$ if $w(a b)>\theta$.

Threshold Physarum-graphs extracted from 62 laboratory experiments are shown in Figs. 5 and 6. The graph becomes planar only for $\theta=\frac{9}{62}$ (Figs. 5p), i.e. when edges occurred in over $15 \%$ of the experiments. We can therefore infer that the Physarum graph is planar. However, with acquiring planarity 


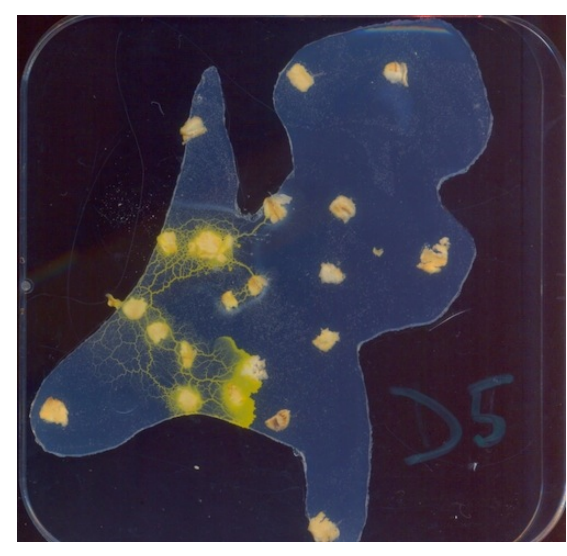

(a) $t=22 \mathrm{~h}$

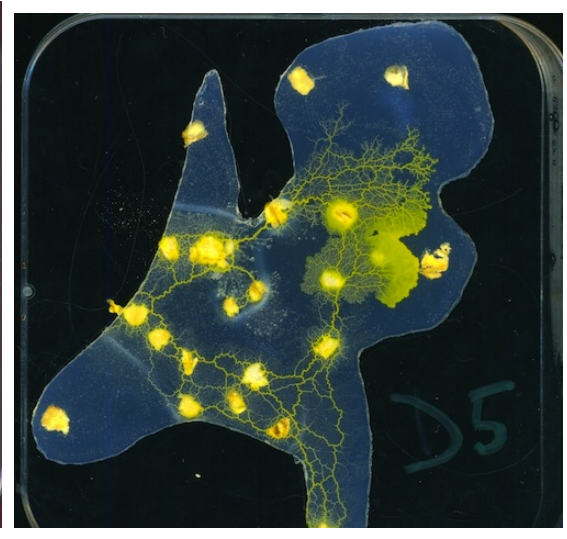

(b) $t=43 \mathrm{~h}$

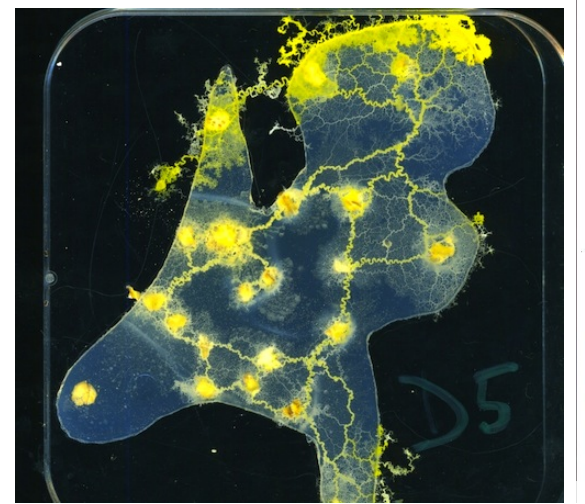

(c) $t=65 \mathrm{~h}$

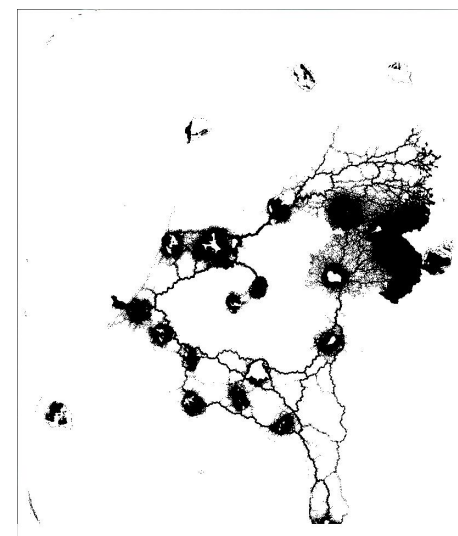

(e) $t=43 \mathrm{~h}$ (d) $t=22 \mathrm{~h}$
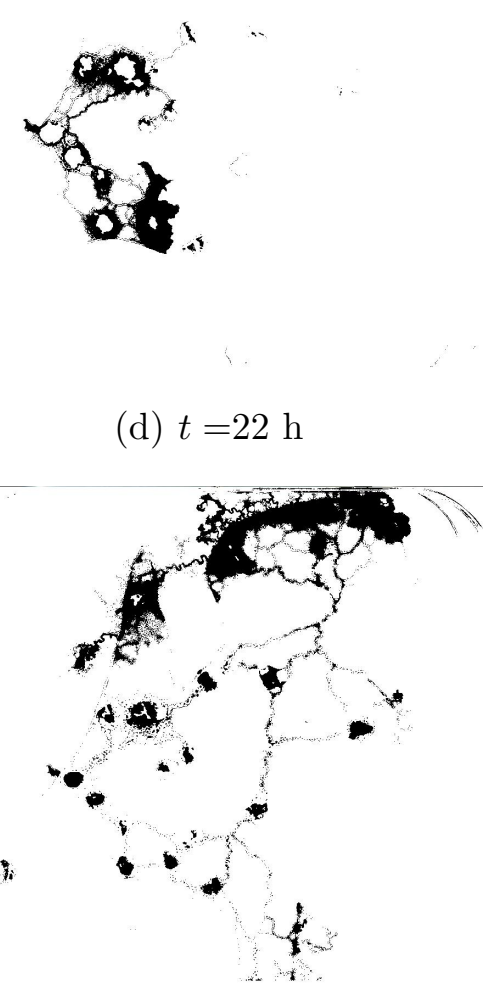

(f) $t=65 \mathrm{~h}$

Fig. 5. Plasmodium does not always span all cities (sources of food): (a)-(d) scanned image of experimental Petri dish. Time elapsed from inoculation is shown in the sub-figure captions. (e)-(h) binary images, $\Theta=(100,100,100)$. 


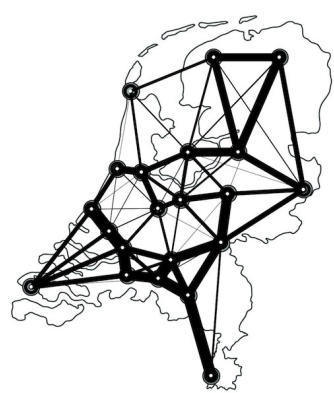

(g) $\theta=0$

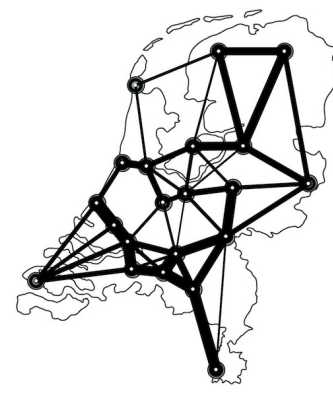

(k) $\theta=\frac{4}{62}$

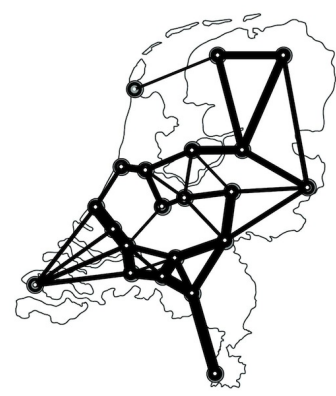

(o) $\theta=\frac{8}{62}$

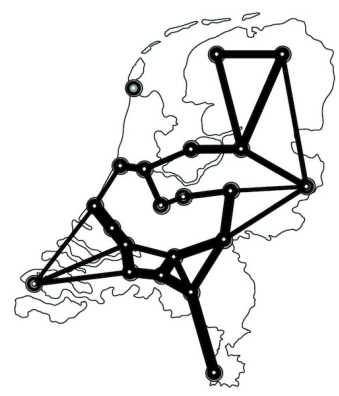

(s) $\theta=\frac{12}{62}$

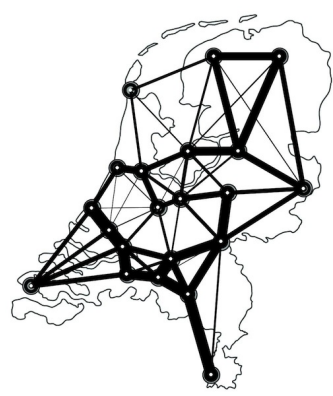

(h) $\theta=\frac{1}{62}$

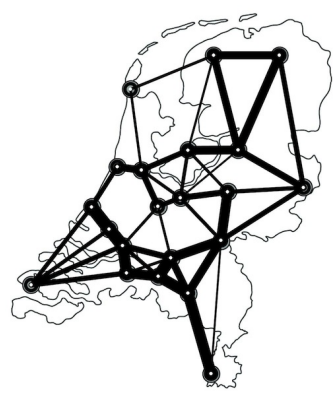

( $\ell$ ) $\theta=\frac{5}{62}$

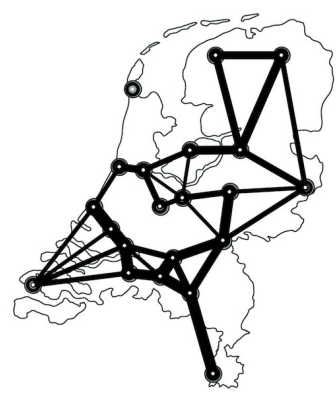

(p) $\theta=\frac{9}{62}$

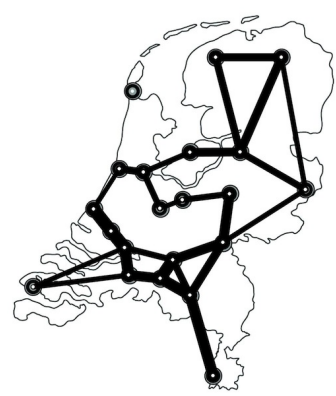

(t) $\theta=\frac{13}{62}$

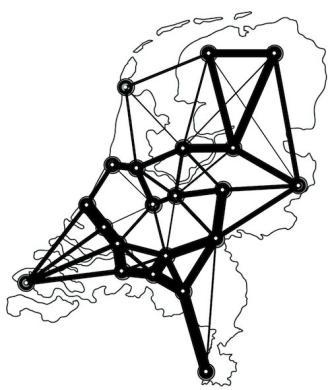

(i) $\theta=\frac{2}{62}$

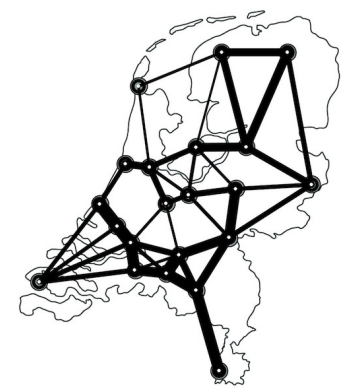

(m) $\theta=\frac{6}{62}$

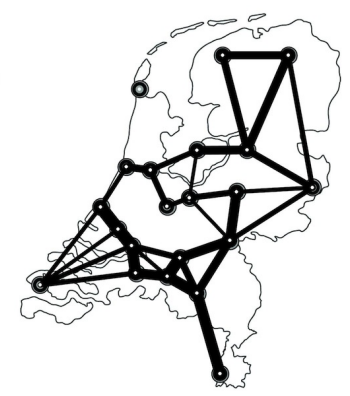

(q) $\theta=\frac{10}{62}$

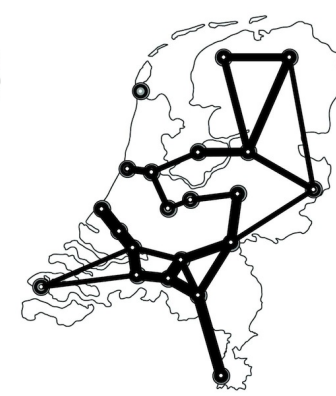

(u) $\theta=\frac{14}{62}$

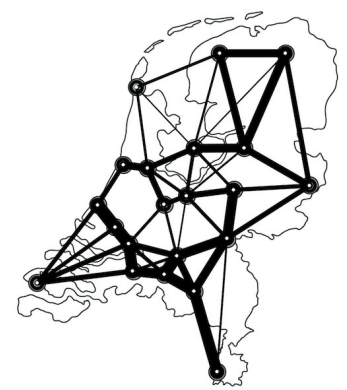

(j) $\theta=\frac{3}{62}$

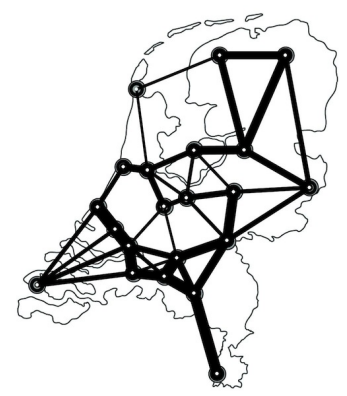

(n) $\theta=\frac{7}{62}$

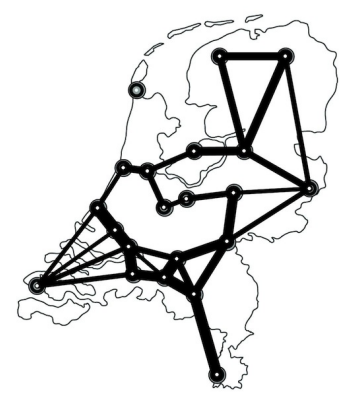

(r) $\theta=\frac{11}{62}$

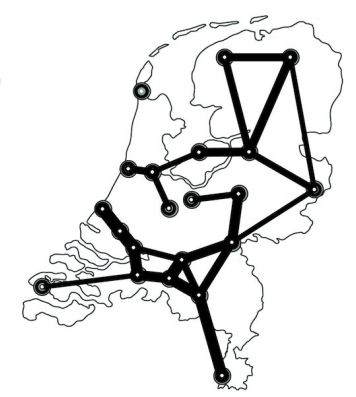

(v) $\theta=\frac{15}{62}$

Fig. 5. Configurations of threshold Physarum-graph $\mathbf{P}(\theta)$ for $\theta=0, \frac{1}{62}, \ldots, \frac{15}{62}$ Thickness of an edge is proportional to the edge's weight. 


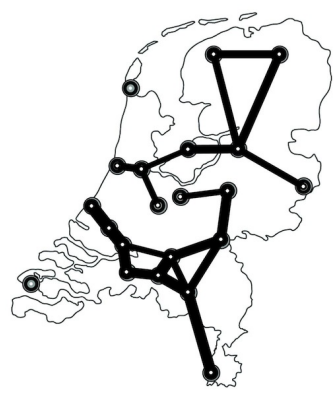

(a) $\theta=\frac{16}{62}$

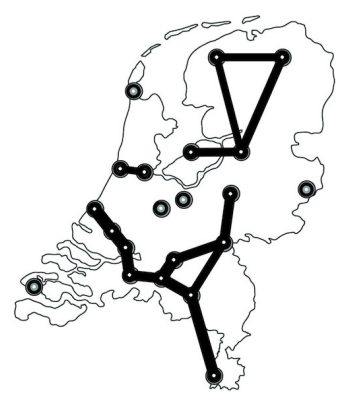

(e) $\theta=\frac{22}{62}$

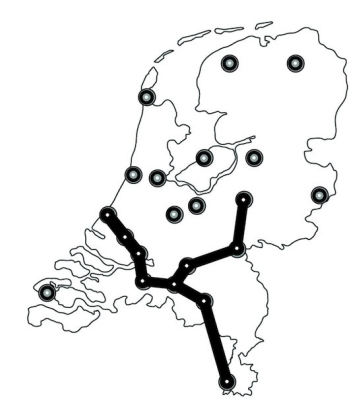

(i) $\theta=\frac{27}{62}$

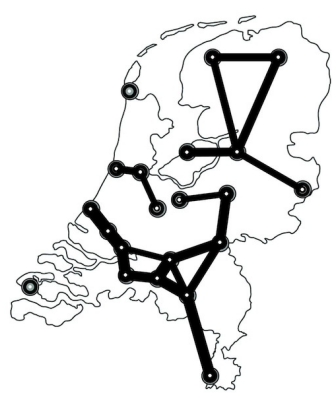

(b) $\theta=\frac{17}{62}$

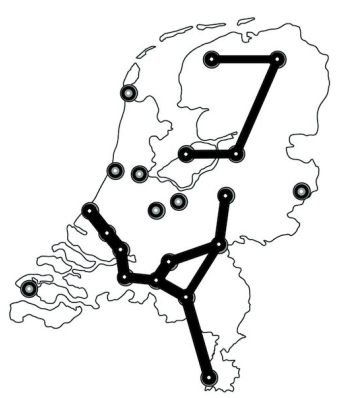

(f) $\theta=\frac{23}{62}$

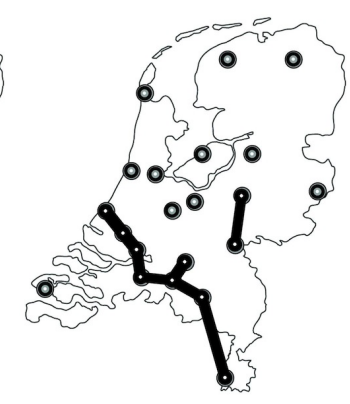

(j) $\theta=\frac{30}{62}$

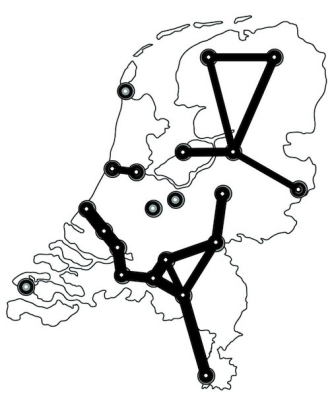

(c) $\theta=\frac{19}{62}$

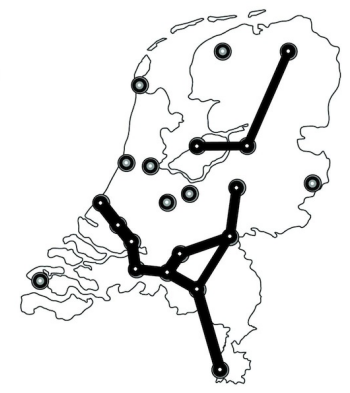

(g) $\theta=\frac{25}{62}$

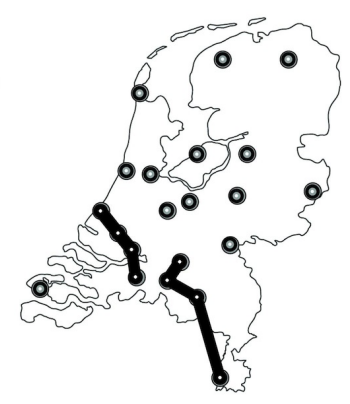

(k) $\theta=\frac{31}{62}$

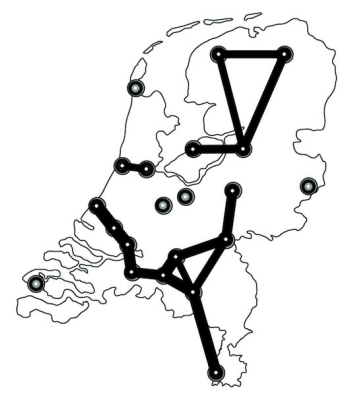

(d) $\theta=\frac{21}{62}$

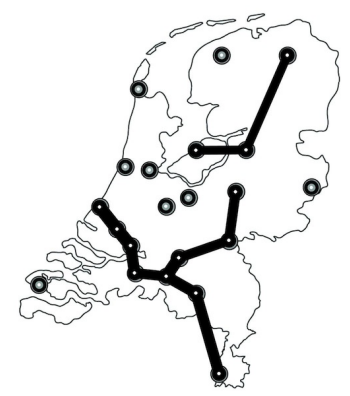

(h) $\theta=\frac{26}{62}$

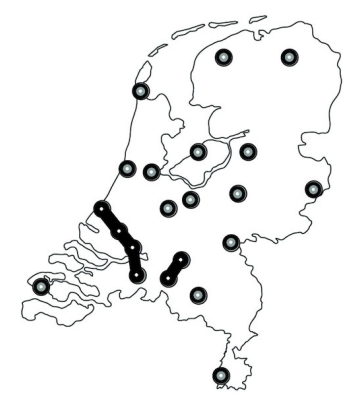

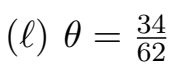

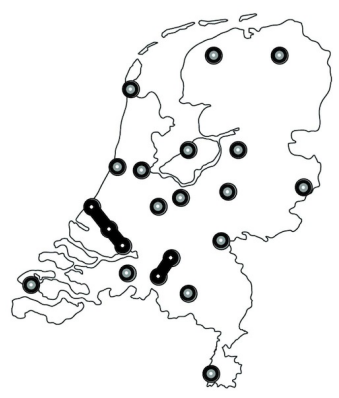

(m) $\theta=\frac{35}{62}$

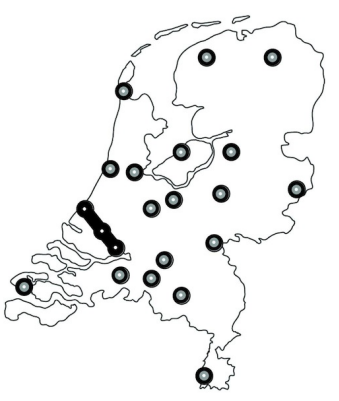

(n) $\theta=\frac{37}{62}$

Fig. 6. Configurations of threshold Physarum-graph $\mathbf{P}(\theta)$ for $\theta=\frac{16}{62}, \frac{17}{62}, \ldots, \frac{37}{62}$ Thickness of an edge is proportional to the edge's weight. 
the graph becomes disconnected: one node, Den Helder city, becomes isolated.

The Physarum-graphs become acyclic for $\theta=\frac{26}{62}$ (Fig. 6h), i.e., when its edges appear as protoplasmic tubes in over $41 \%$ of the experiments. When the graph becomes acyclic it is split into a set of isolated nodes: Den Helder, Leeuwarden, Haarlem, Amsterdam, Utrecht, Amersfoort, Enschede, Middelburg, and two additional components. One component is a chain of three cities: Lelystad, Zwolle and Groningen. The second component is a tree routed in Tilburg. The tree has three linear branches:

- Tilburg - Breda - Dordrecht - Rotterdam - Den Haag

- Tilburg - Hertogenbosch - Nijmegen - Apeldoon

- Tilburg - Eindhoven - Maastricht.

This tree is a characteristic feature of the Physarum-graph and it appears in over $60 \%$ of experiments. The tree is 'destroyed' when $\theta \geq \frac{30}{62}$ (Fig. 6j), then only chains remain, which give away isolated nodes with further increase of $\theta$. Some chains are more stable then others. Thus, the chain Breda - Dordrecht - Rotterdam - Den Haag appears in over $54 \%$ of experiments (Fig. 6[). While the chain Dordrecht - Rotterdam - Den Haag appears in almost $60 \%$ of experiments.

The experiments have now provided a reasonably consistent set of connections between the various urban centres in the Netherlands. The next question is to asses how well these Physarum graphs approximate the Netherlands motorway network. A graph $\mathbf{H}$ of Dutch motorways is Fig. 7 a. We construct the motorway graph $\mathbf{H}$ as follows. Let $\mathbf{U}$ be a set of urban regions/cities, for any two regions $a$ and $b$ from $\mathbf{U}$, the nodes $a$ and $b$ are connected by an edge $(a b)$ if there is a motorway starting in vicinity of $a$ and passing in vicinity of $b$ and not passing in vicinity of any other urban area $c \in \mathbf{U}$. If there is a branching motorways, which e.g. starts in $a$ goes in the direction of $b$ and $c$ and at some point branches towards $b$ and $c$, we then add two separate edges $(a b)$ and $(a c)$ to the graph $\mathbf{H}$.

The intersection $\mathbf{P}(\theta) \cap \mathbf{H}$ of Physarum and motorways graphs is shown in Fig. 7 bcd for $\theta=0, \frac{8}{16}$ and $\frac{15}{16}$. A relaxed probabilistic Physarum graph $\mathbf{P}(0)$, where an edge appears in the graph if it is recorded in at least one experiment, matches the motorway graph $\mathbf{H}$ almost perfectly. Just three edges of $\mathbf{H}$ are not presented in $\mathbf{P}(0) \cap \mathbf{H}$ :

- (Amsterdam, Der Helden),

- (Zwolle, Apeldoon),

- (Rotterdam, Dordrecht) (Fig. 7b).

$\theta=\frac{8}{16}$ is the highest value for which Physarum graph $\mathbf{P}(\theta)$ remains connected (Fig. 5). Graph $\mathbf{P}\left(\frac{8}{62}\right) \cap \mathbf{H}$ loses few more edges (presented in $\mathbf{P}\left(\frac{0}{62}\right) \cap \mathbf{H}$ : 


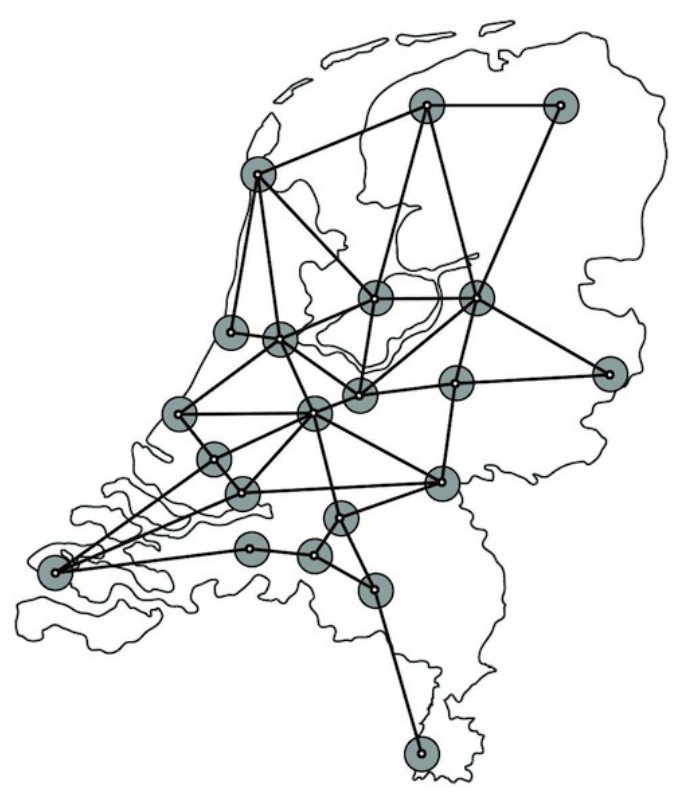

(a) $\mathbf{H}$

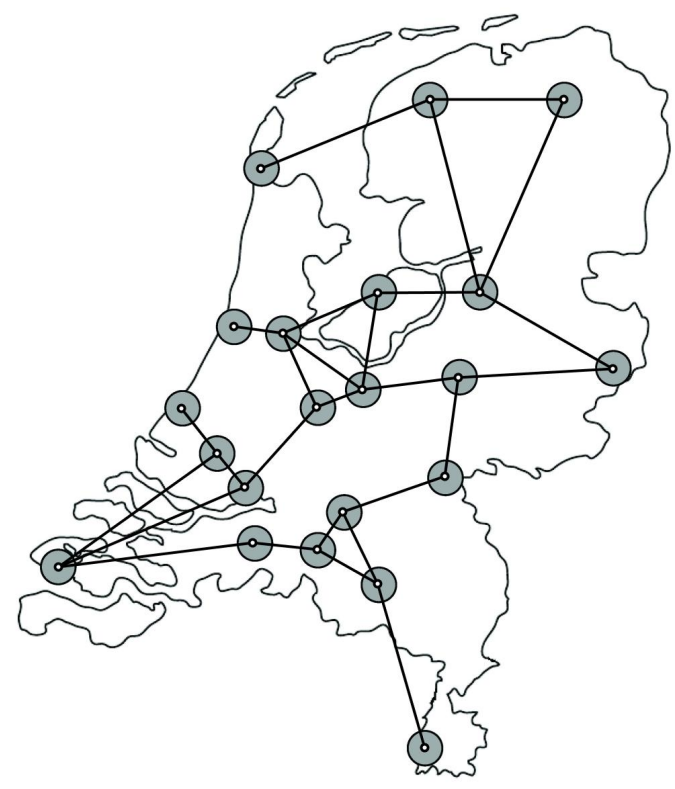

(c) $\mathbf{P}\left(\frac{8}{62}\right) \bigcap \mathbf{H}$

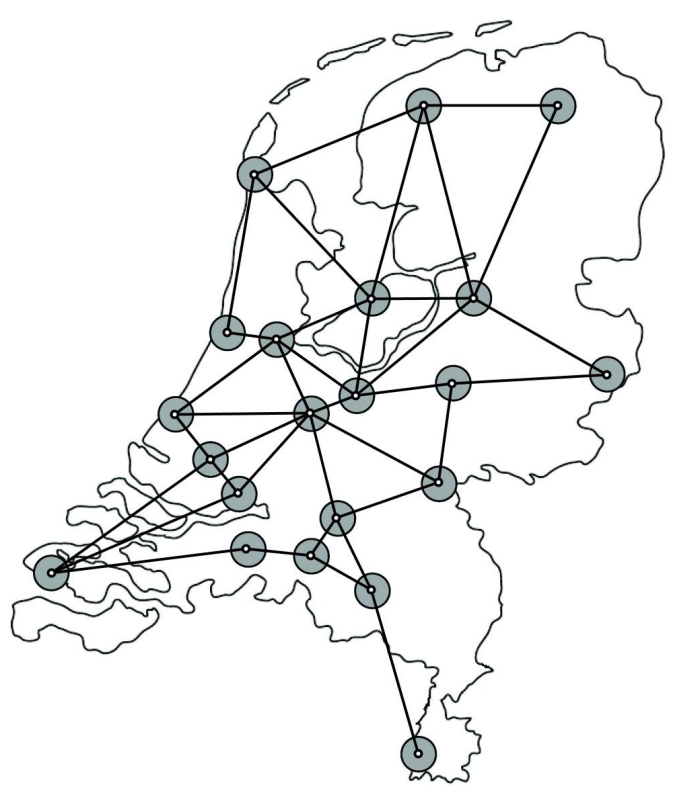

(b) $\mathbf{P}(0) \bigcap \mathbf{H}$

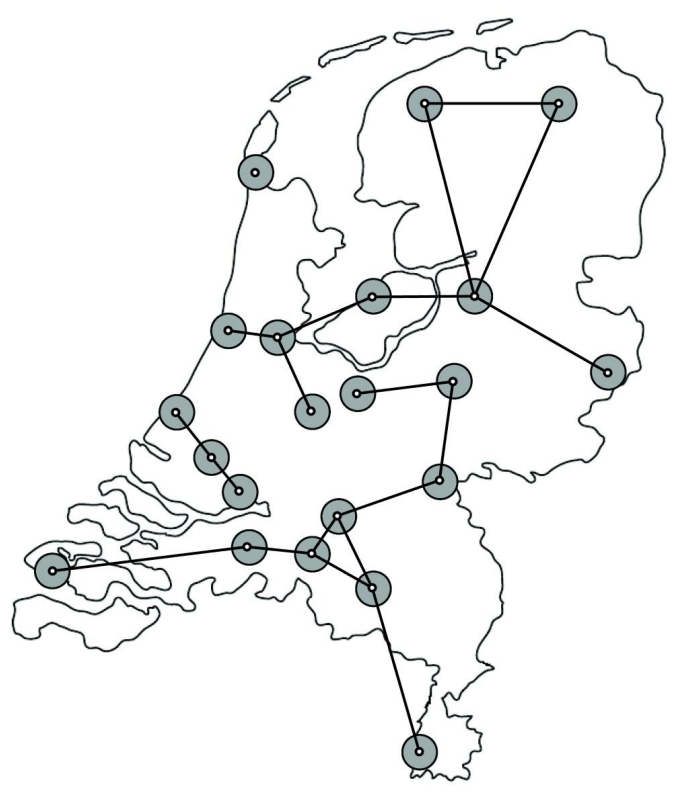

(d) $\mathbf{P}\left(\frac{15}{62}\right) \bigcap \mathbf{H}$

Fig. 7. Graph $\mathbf{H}$ of man-made motorway network is shown in (a). Intersection $\mathbf{P}(\theta) \cap \mathbf{H}, \theta=1, \frac{8}{16}$ and $\frac{15}{16}$, of Physarum $\mathbf{P}$ and motorways $\mathbf{H}$ graphs is shown in (bcd).

- (Den Helder, Lelystad),

- (Den Helder, Haarlem),

- (Leeuwarden, Lelystad),

- (Utrecht, Dordrecht),

- (Utrecht, Nijmegen), 
- (Breda, Enschede),

- (Utrecht, Enschede) (Fig. 7r).

As soon as $\theta$ reaches value $\frac{15}{16}$ the graph $\mathbf{P}(\theta) \cap \mathbf{H}$ becomes separated on isolated node Den Helder, and there components:

- chain Enschede - Den Haag -Rotterdam,

- cycle Nijmegen - Breda - Middelburg with branches Breda - Hertogenbosch - Tilburg, Middelburg - Eindhoven and Nijmegen - Dordrecht - Apeldoon Amersfoort,

- cycle Leeuwarden - Groningen - Zwolle with branches Zwolle - Enschede and Zwolle - Lelystad - Amsterdam - Haarlem, Utrecht (Fig. 7d).

\section{Comparing motorway and Physarum graphs with proximity graphs}

We conjectured that plasmodium of Physarum polycephalum constructs planar proximity graphs by its protoplasmic network [5]. A protoplasmic network constructed in any particular experiment is planar, a generalised Physarum graph $\mathbf{P}$ may be non-planar. A planar graph consists of nodes which are points on a Euclidean plane with edges which are straight segments connecting the points. A planar proximity graph is a planar graph where two points are connected by an edge if they are close in some sense. Usually a pair of points are assigned a certain neighborhood, and the pair are connected by an edge if their neighborhood is empty. Relative neighborhood graph [14, Gabriel graph [23], $\beta$-skeletons [16] and spanning tree are most known examples of proximity graphs.

For self-consistency we provide a brief definition of the following graphs:

- RNG: Points $a$ and $b$ are connected by an edge in RNG if no other point $c$ is closer to $a$ and $b$ than $\operatorname{dist}(a, b)$ [28].

- GG: Points $a$ and $b$ are connected by an edge in GG if a disc with diameter $\operatorname{dist}(a, b)$ centered in middle of the segment $a b$ is empty [13]23].

- $\mathbf{B S}(\beta)$ : A $\beta$-skeleton, $\beta \geq 1$, is a planar proximity undirected graph of an Euclidean point set where nodes are connected by an edge if their lunebased neighborhood contains no other points of the given set; parameter $\beta$ determines size and shape of the nodes' neighborhoods [16].

- MST: The Euclidean minimal spanning tree (MST) [22] is a connected acyclic graph which has minimal possible sum of edges' lengths.

The graphs are related as $\mathbf{M S T} \subseteq \mathbf{R N G} \subseteq \mathbf{B S} \subseteq \mathbf{G G}[28,23[14]$.

We constructed a relative neighbourhood graph [28] RNG (Fig. 83), a Gabriel 


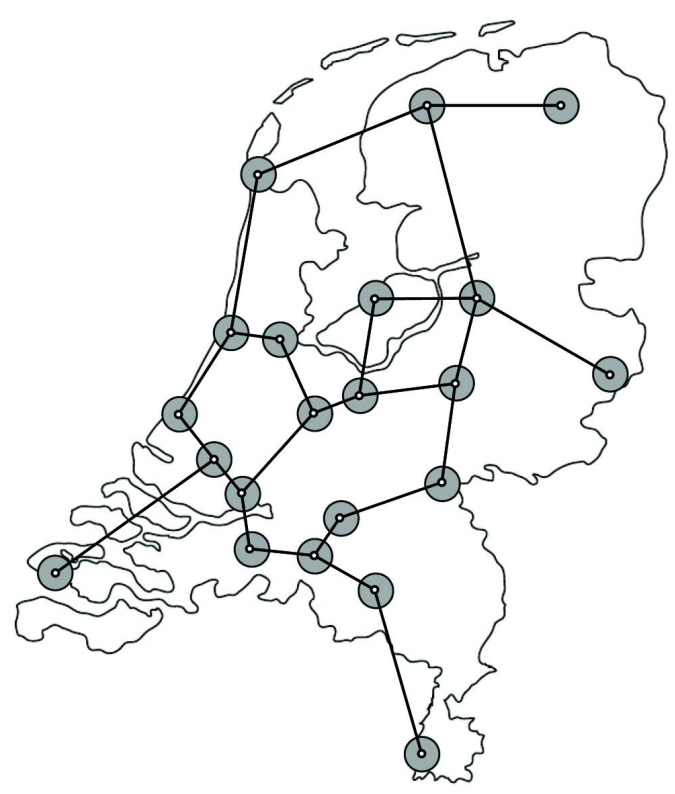

(a) RNG

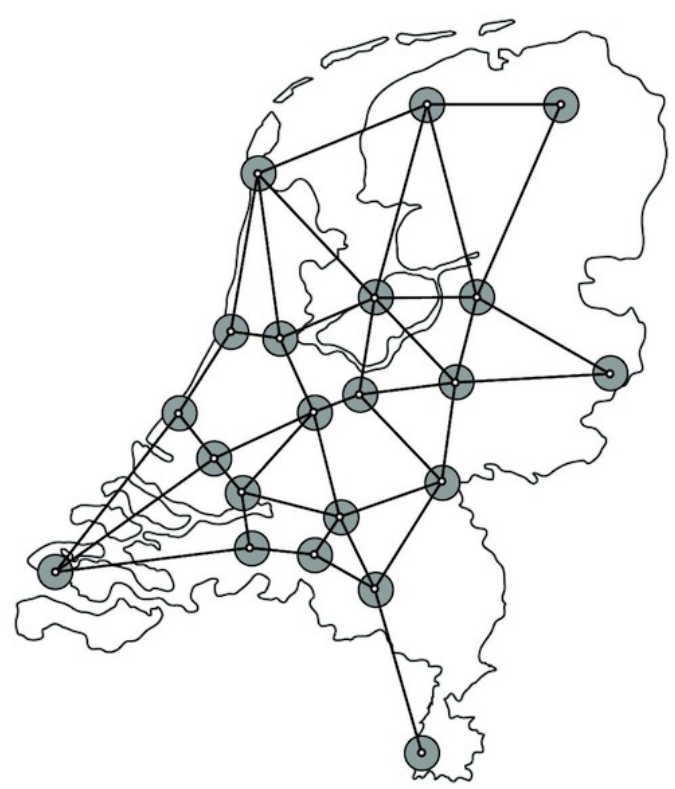

(c) $\mathbf{G G}$

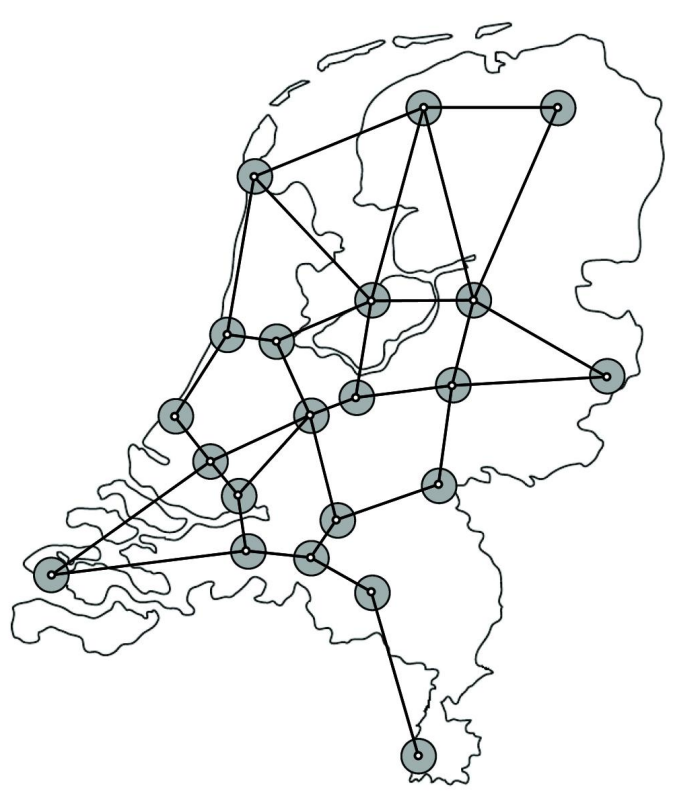

(b) $\mathbf{B S}(1.5)$

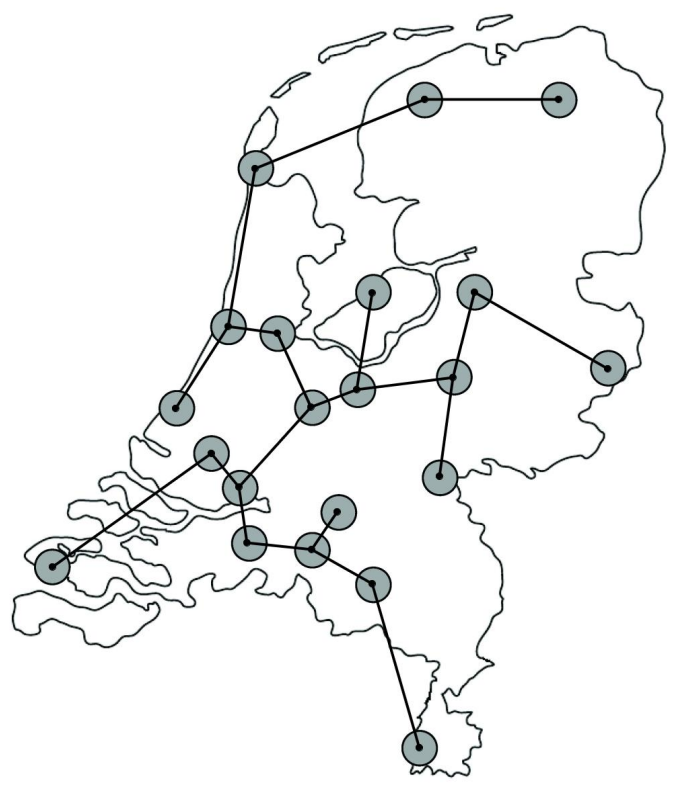

(d) MST

Fig. 8. Proximity graphs constructed on regions $\mathbf{U}$ : (a) relative neighbourhood graph RNG, (b) $\beta$-skeleton with control parameter 1.5, BS(1.5) (c) Gabriel graph GG, (d) Minimum spanning tree MST

graph [13,23] GG (Fig. 8c), $\beta$-skeleton ((Fig. 8b) and a minimum spanning tree MST (Fig. 8d) (we rooted MST in Amsterdam) over nodes corresponding to centres of urban areas. We then calculated intersections of these graphs with the Physarum graph $\mathbf{P}(0)$ and the motorway graph $\mathbf{H}$, see Fig. 9. 


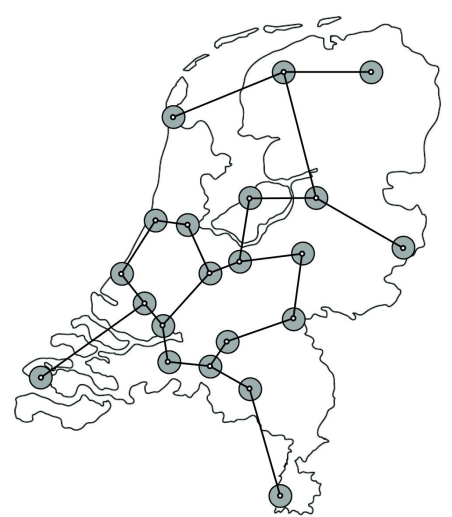

(a) $\mathbf{P} \cap \mathbf{R N G}$

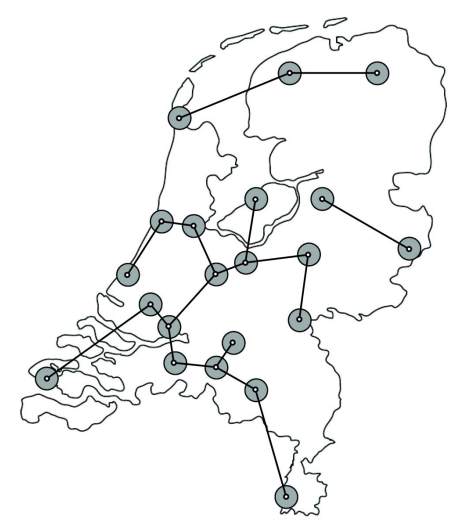

(d) $\mathbf{P} \cap$ MST

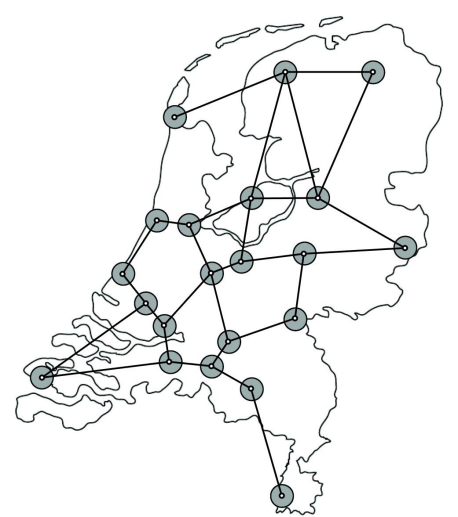

(b) $\mathbf{P} \cap \mathbf{B S}(1.5)$

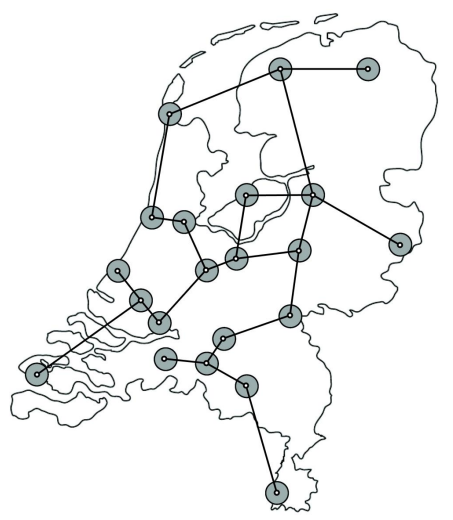

(e) $\mathbf{H} \bigcap \mathbf{R N G}$

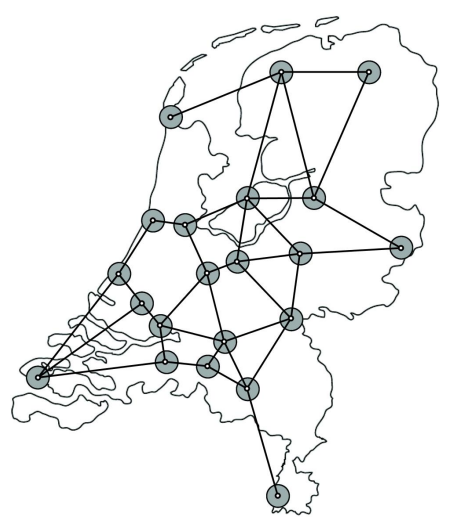

(c) $\mathbf{P} \cap \mathbf{G G}$

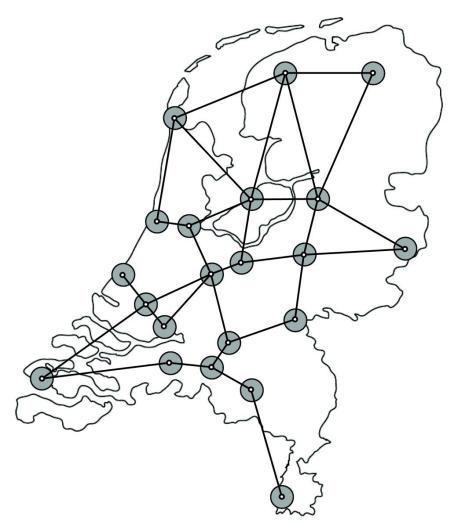

(f) $\mathbf{H} \bigcap \mathbf{B S}(1.5)$

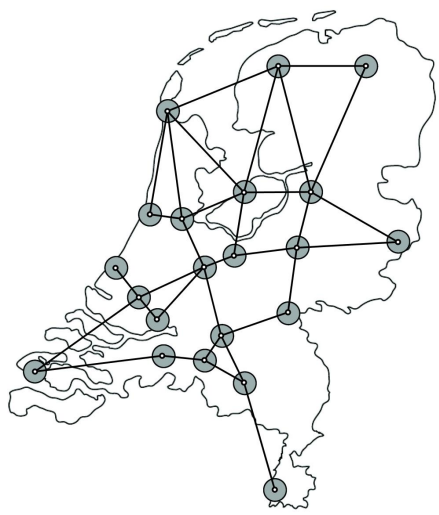

(g) $\mathbf{H} \cap \mathbf{G G}$

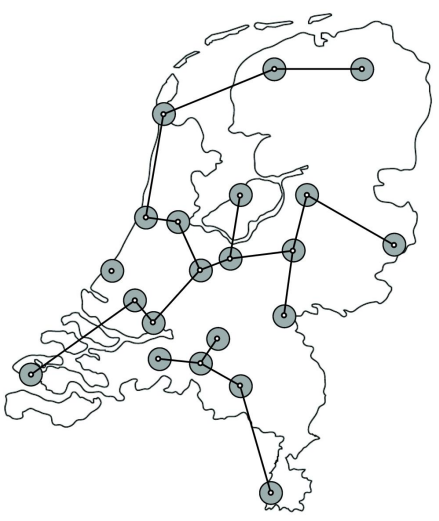

(h) $\mathbf{H} \cap \mathrm{MST}$

Fig. 9. Intersection of Physarum graph $\mathbf{P}(0)$ (a)-(d) and motorway graph $\mathbf{H}(\mathrm{e})-(\mathrm{h})$ with proximity relative neighbourhood graph $\mathbf{R N G}, \beta$-skeleton $\mathbf{B}(1.5)$, Gabriel graph GG and minimum spanning tree MST.

The following is a list of edges of the proximity graphs that are not present in Physarum or motorway graphs:

- $\mathbf{P} \cap \mathbf{M S T}=\mathbf{M S T}-\{($ Den Helder, Haarlem $),($ Zwolle, Apeldoon $)\}$ 
- $\mathbf{P} \cap \mathbf{R N G}=\mathbf{R N G}-\{($ Den Helder, Haarlem $),($ Zwolle, Apeldoon $)\}$

- $\mathbf{P} \cap \mathbf{B S}(1.5)=\mathbf{B S}(1.5)-\{($ Den Helder, Haarlem), (Zwolle, Apeldoon), (Utrecht, Den Haag)\}

- $\mathbf{P} \cap \mathbf{G G}=\mathbf{G G}-\{($ Den Helder, Haarlem), (Zwolle, Apeldoon), (Utrecht, Den Haag), (Den Helder, Amsterdam), (Den Helder, Lelystad)\}

- $\mathbf{H} \cap \mathbf{M S T}=\mathbf{M S T}-\{($ Haarlem, Enschede), (Rotterdam, Hertogenbosch $)\}$

- $\mathbf{H} \cap \mathbf{R N G}=\mathbf{R N G}-\{($ Haarlem, Enschede), (Rotterdam, Hertogenbosch $)\}$

- $\mathbf{H} \cap \mathbf{B S}(1.5)=\mathbf{B S}(1.5)-\{($ Haarlem, Enschede), (Rotterdam, Hertogenbosch) $\}$

- $\mathbf{H} \cap \mathbf{G G}=\mathbf{G G}-\{($ Haarlem, Enschede), (Rotterdam, Hertogenbosch), (Enschede, Tilburg), (Rotterdam, Nijmegen), (Amersfoort, Dordrecht), (Dordrecht, Tilburg)\}.

The motorway graph closely matches the spanning tree, relative neighbourhood graph and $\beta$-skeleton. Only two edges (Haarlem, Enschede), (Rotterdam, Hertogenbosch), presented in RNG, MST, BS(1.5) do not exist in $\mathbf{H}$. The fact that the relative neighbourhood graph is almost a sub-graph of the motorway graph indicates intrinsically logical organization of the transport networks in the Netherlands. This is because a relative neighbourhood graph is commonly considered to be optimal in terms of total edge length and travel distance, and is known to be a good approximation of road networks [30,31].

Said that the transport networks in the Netherlands are redundant - from slime mould's point of view - because there is a substantial number of edges of $\mathbf{H}$ not presented in $\mathbf{R N G}$.

The same can be said about the Physarum graph, because only edges (Den Helder, Haarlem) and (Zwolle, Apeldoon) of RNG are not represented by protoplasmic tubes. Under-representations of $\beta$-skeleton and GG in between $\mathbf{P}(0)$ are much more substantial: three and five edges, respectively.

\section{$5 \quad$ Flooding}

In this section we decribe the experiments which investigate the capability of the plasmodium to adapt the its network during induced flooding of the petri-dish. In essence we investigate how the plasmodium would calculate and adapt the transport network, if the Netherlands were to suffer similar flooding of its cities and roads. Experiments on flooding were conducted in $12 \times 12 \mathrm{~cm}$ Petri dishes. A Petri dish was raised by $1-2 \mathrm{~cm}$ in its south-east corner and partly filled with liquid (distilled water, either pure or coloured with one drop of food coloring) (Fig. 10a). In most cases the flooded area included Middelburg on the south-west and Groningen on the north-east. In the central part of the country the flooding often reached Zwolle. The exact flooded area varied between experiemtns due to slight variations in the thickness of agar gel 


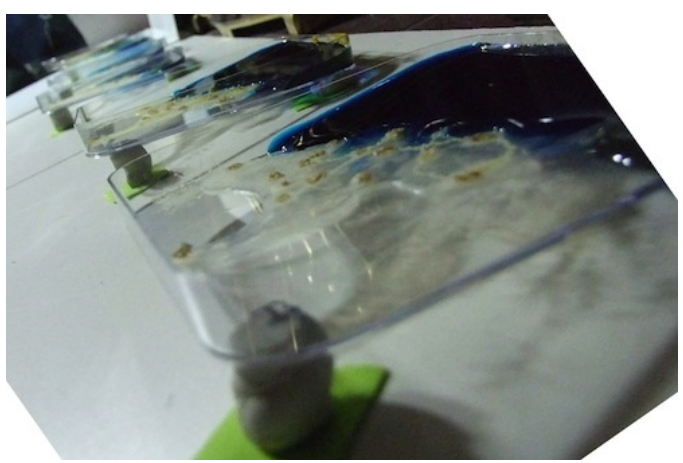

(a)

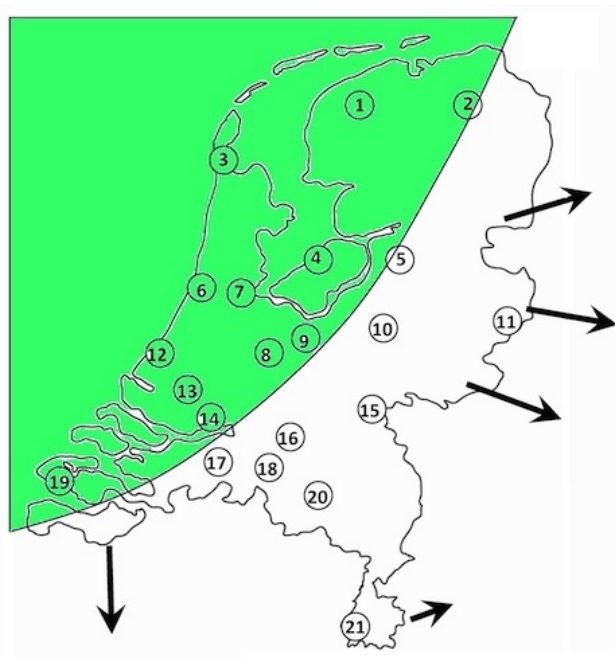

(b)

Fig. 10. Flooding setup: (a) array of Petri dishes during imitated flooding, (b) Flooding scheme. Flooded part is filled is shaded (green), direction of outward migration are shown by arrows, each large arrow represents $20 \%$, while small arrow $10 \%$.

substrate and minor differences in inclinations of Petri dishes, the flooding scheme shown in (Fig. 10) is rather indicative.

Initially plasmodium reacts to flooding with increased activity. During the first few hours of flooding the plasmodium typically increases its branching at the boundary of the flooded area (Fig. 11a). Often there are indications of indiscriminate increase of foraging, panic foraging. For example, in Fig. 11b we can see active sprawling of plasmodium in the areas around Apeldoon, Dordrecht and Enschede: no protoplasmic tubes are formed but rather uniform sheets of plasmodium propagate in these areas. Eventually the activity ceases and flooded transport links becomes abandoned (Fig. 11k).

In some cases no 'panic' branching occurs, but non-flooded protoplasmic tubes become thicker due to increased transport of nutrients and relocation of masses of protoplasm from areas affected by flooding (Fig. 12 a). Often only protoplasmic tubes located along flood line are hypertrophied, for example there is an increased thickness of tubes along the route Hertogenbosch - Apeldoon Zwolle - Groningen in Fig. 12b.

With time, water is absorbed by the gel and is sucked under the gel plate due to capillary forces, which in turn causes the overall humidity to increase. This is associated with a reduced concentration of nutrients, and an increased concentration of metabolites ejected in the agar plate, force the plasmodium to abandon the agar plate and migrate beyond. Often the plasmodium attempts to complete evacuation by crawling onto the water surface (for example see Fig. 13). 


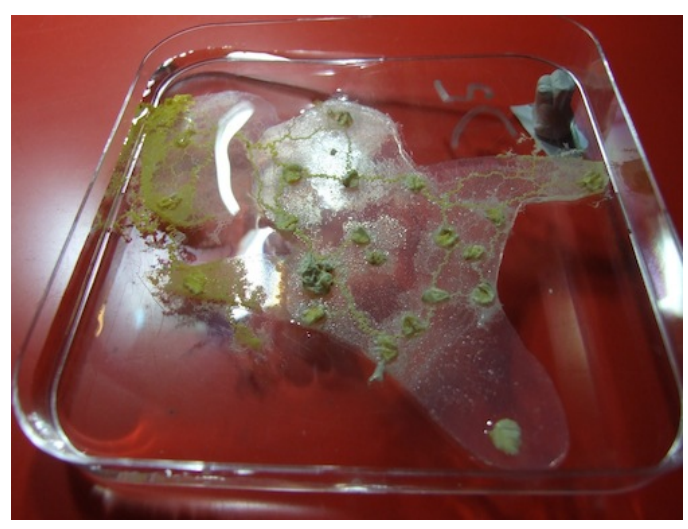

(a) $t=0 \mathrm{~h}$

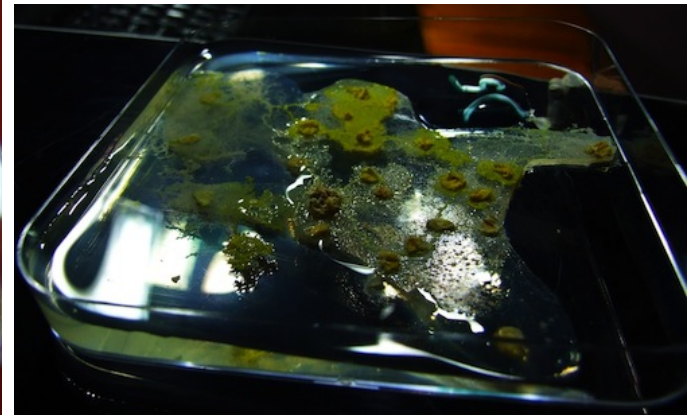

(b) $t=30 \mathrm{~h}$

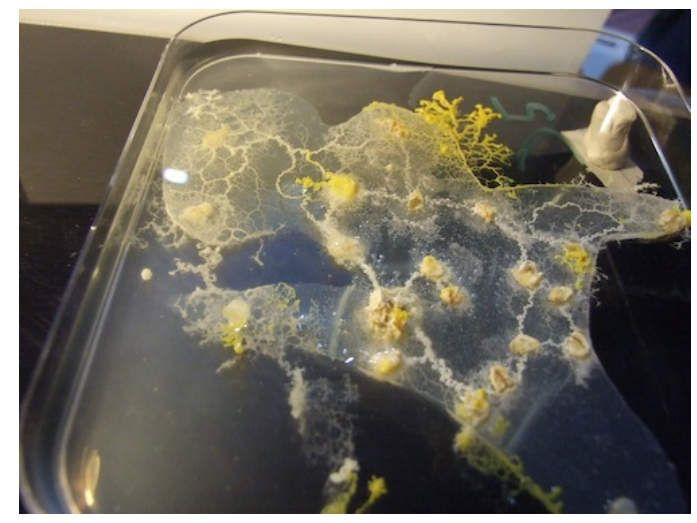

(c) $t=46 \mathrm{~h}$

Fig. 11. Illustration of plasmodium behaviour during experiment on flooding. Photographs are made at different angles.

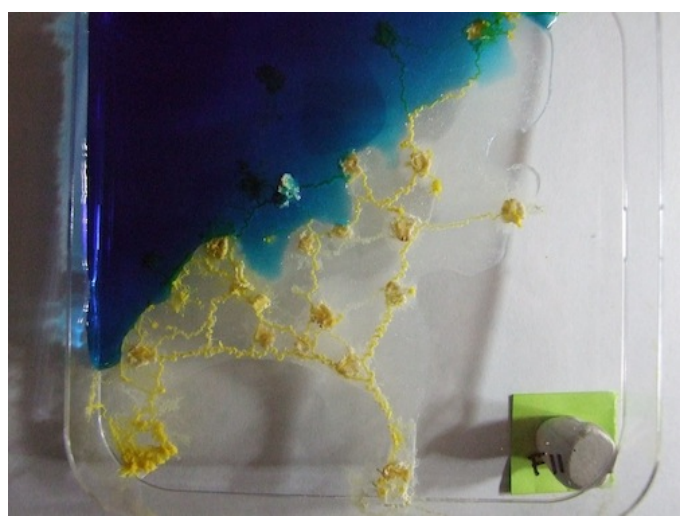

(a)

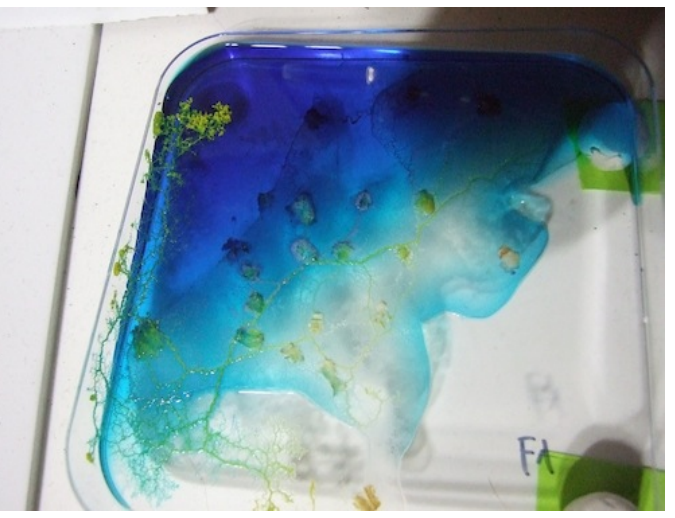

(b)

Fig. 12. Compensation of transport networks (a) and increase in borderline transport (b). 


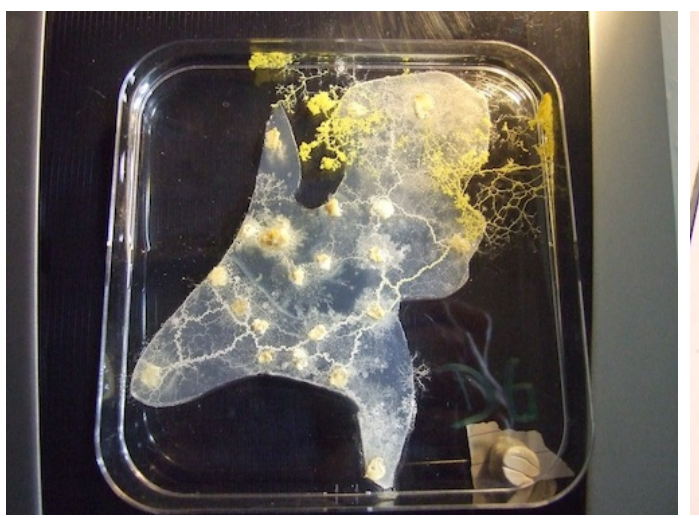

(a)

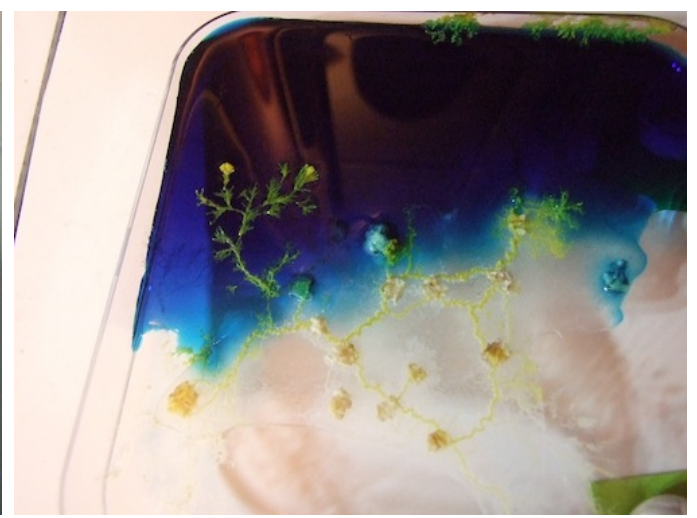

(b)

Fig. 13. Examples of evacuation.

Outward migration, calculated in nine experiments, are shown in Fig. 10b. We conclude that if a flooding of the Netherlands were to happen the major impact of migration (60\%) will be felt by Western Germany, with just a minor impact on Northen France (20\%) and a very slight impact on Belgium (10\%). If the Netherlands were flooded the Western Germany accepts the largest impact of mass-migration.

\section{Discussion}

In laboratory experiments with plasmodium of Physarum polycephalum we discovered that the Physarum protoplasmic network forms a sub-network of man-made motorway networks, i.e. every transport link represented by Physarum can also be found as a segment of the motorway network. However, the converse does not hold. The motorway network is not a sub-graph of the Physarum network; there are edges of motorway graph not represented by edges Physarum graph. Transport links Amsterdam to Der Helden, Zwolle to Apeldoon, and Rotterdam to Dordrecht are never presented in Physarum graphs. This may be interpreted as if the man-made motorway network in the Netherlands is redundant from Physarum point of view.

Also, in many experiments Den Helder city remains disconnected from other cities. This is either a mishap of the Physarum approach, e.g. plasmodium prefers not to enter narrow peninsula of North Holland, or an indication of a somewhat inefficient location of Den Helder.

The most robust component of the Physarum graph, the component which is present in the majority of experiments is a tree with three linear branches. First branch is Tilburg - Breda - Dordrecht - Rotterdam - Den Haag, second is Tilburg - Hertogenbosch - Nijmegen - Apeldoon, and third is Tilburg 
- Eindhoven - Maastricht. A possible explanation would be that relative positions and distances between the cities in this trees is optimal for plasmodium physiological functioning. Cities are not close enough to be contaminated by products of plasmodium activity but close enough not to put significant strain on the pumping of nutrients between distant parts of plasmodium's body.

If we prune the Physarum graph by removing edges which occur in less thana quarter of experiments and look at the intersection (i.e. the set of edges present in both graphs) of this graph with a graph of motorways we find that intersection consist of three disconnected components. The first component is a chain Enschede - Den Haag - Rotterdam. The second component is a cycle Nijmegen - Breda - Middelburg with branches Breda - Hertogenbosch - Tilburg, Middelburg - Eindhoven and Nijmegen - Dordrecht - Apeldoon - Amersfoort. FInally, the third component is a cycle Leeuwarden - Groningen - Zwolle with branches Zwolle - Enschede and Zwolle - Lelystad - Amsterdam - Haarlem, Utrecht. With respect to the proximity graph the key finding is that relative neighbourhood graphs (which is commonly recognised as a best approximation of urban streets and transport networks) are almost (apart of two edges) subgraphs of the Physarum graph and motorway graph.

By physically imitating flooding of some parts of the Netherlands we predicted that if a real flooding were to occur, the following events will take place: substantial increase in traffic on the parts of motorway networks close to the boundary between flooded and non-flooded areas, propagation of the traffic congestion to all non-flooded parts of the country, complete paralysis and abandonment of transport network, migration of population from the Netherlands to Germany and France, and Belgium.

The plasmodium is incredibly similar, esp. in wave-like behaviour, to subexcitable non-linear media [2] and is mainly guided by gradients of chemoattractants [6]. Based on these two facts we could assume the plasmodium must spread from Amsterdam omni-directionally and then start forming branches between cities, as e.g. illustrated in Fig. 14. Such phenomena do not occur, rather the plasmodium behaves more like a 'single-headed' creature, it chooses one direction of movement, explores it, then chooses another direction and explores it again, see Fig. 3. Such behaviour of the plasmodium may explain the differences between ideal planar proximity graphs and protoplasmic networks constructed by the plasmodium.

In future work we plan to undertake more experiments on Physarum-based imitation of road formation in other European countries, and may be even make experiments at a large scale, where plasmodium grows over the whole Europe. We also plan to evaluate the city state of Singapore, this present a fairly unique road network that has been carefully planned and constructed within the last 50 years. One further objective would be to try and incorporate 


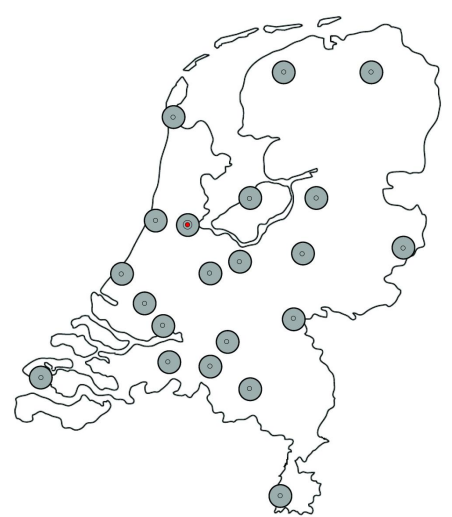

(a) $t=1$

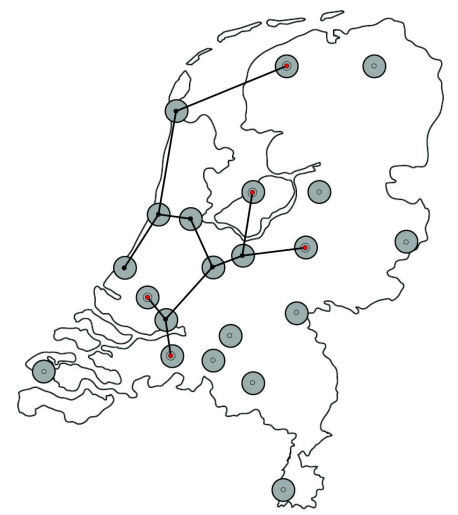

(d) $t=4$

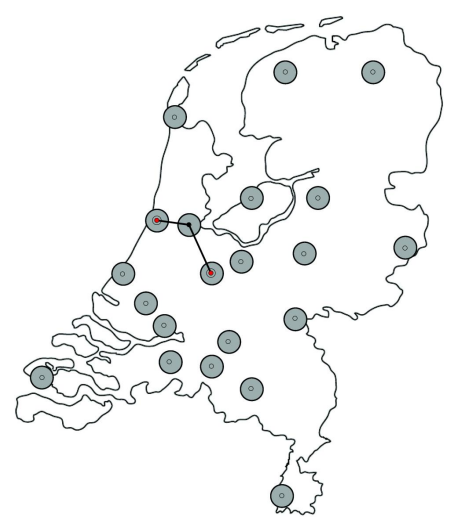

(b) $t=2$

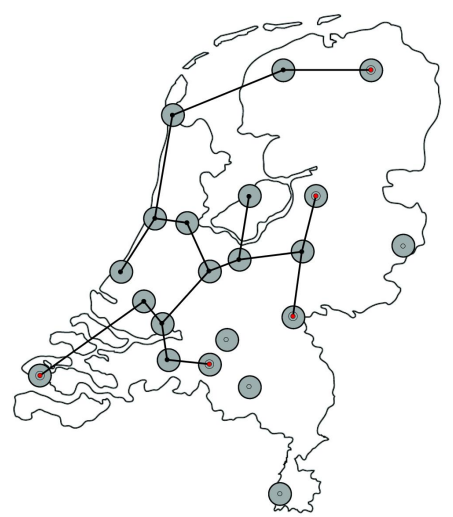

(e) $t=5$

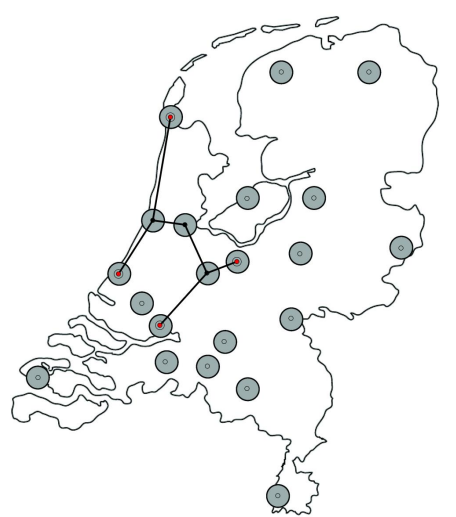

(c) $t=3$

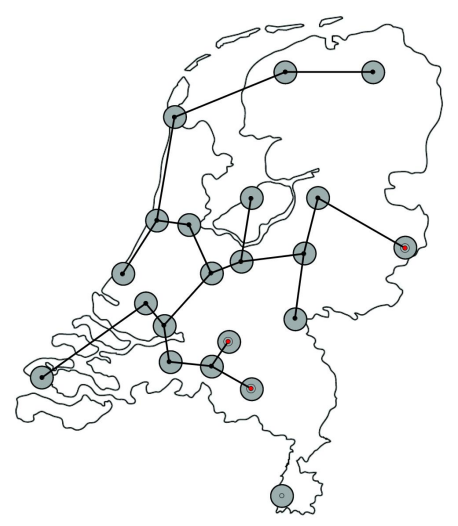

(f) $t=7$

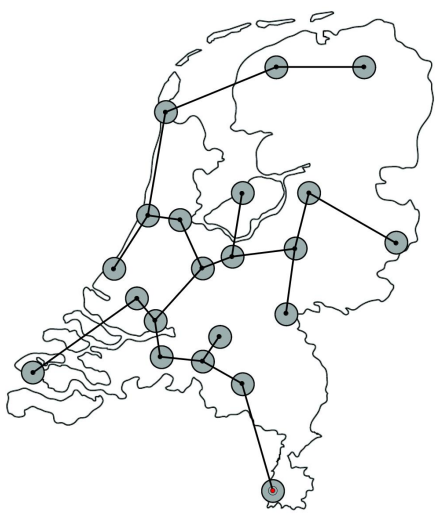

(g) $t=8$

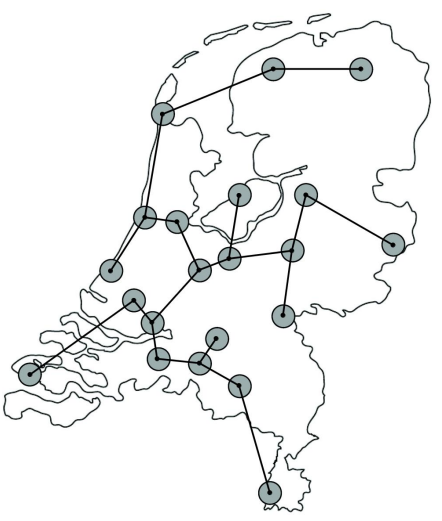

(h) $t=9$

Fig. 14. Snapshots of a growing spanning tree of $\mathbf{U}$ rooted in Amsterdam. Vertices active at time step $t^{\prime}$ are shown by black disc at snapshot $t=t^{\prime}$.

three dimensional landscape elevation into our laboratory experiments. ' 


\section{Acknowledgements}

Peter M.A. Sloot would like to acknowledge the support of this work by the Russian Federation Leading Scientist Grant, contract: 11.G34.31.0019 and the European Union Dynanets project: 233847.

\section{References}

[1] Adamatzky A., De Lacy Costello B., Asai T. Reaction-Diffusion Computers, Elsevier, Amsterdam, 2005.

[2] Adamatzky A. Physarum machines: encapsulating reaction-diffusion to compute spanning tree. Naturwisseschaften 94 (2007) 975-980.

[3] Adamatzky A. Physarum machine: implementation of a Kolmogorov-Uspensky machine on a biological substrate. Parallel Processing Letters 17 (2007) 455467.

[4] Adamatzky A. From reaction-diffusion to Physarum computing. Invited talk at Los Alamos Lab workshop "Unconventional Computing: Quo Vadis?" (Santa Fe, NM, March 2007).

[5] Adamatzky A. Developing proximity graphs by Physarum polycephalum: does the plasmodium follow the Toussaint hierarchy? Parallel Processing Letters (2009), in press.

[6] Adamatzky A. If BZ medium did spanning trees these would be the same trees as Physarum built. Physics Letters A (2009), in press.

[7] Adamatzky A. Hot ice computer. Physics Lett A (2009), in press.

[8] Adamatzky A. and Jones J. Road planning with slime mould: If Physarum built motorways it would route M6/M74 through Newcastle Int J Bifurcaton and Chaos (2010), in print. http://arxiv.org/abs/0912.3967

[9] Adamatzky A. Slime mould logical gates: exploring ballistic approach (2010). http://arxiv.org/abs/1005.2301

[10] Adamatzky A. Physarum Machines: Making Computers from Slime Mould (World Scientific, 2010).

[11] Adamatzky A., Martinez G. J., Chapa-Vergara S. V., Asomoza-Palacio R., Stephens C. R. Approximating Mexican highways with slime mould (2010) http://arxiv.org/abs/1010.0557

[12] Dorigo M. and Stutzle T. Ant Colony Optimization MIT Press, 2004.

[13] Gabriel K. R. and R. R. Sokal. A new statistical approach to geographic variation analysis. Systematic Zoology, 18 (1969) 259-278. 
[14] Jaromczyk J. W. and G. T. Toussaint, Relative neighborhood graphs and their relatives. Proc. IEEE 80 (1992) 1502-1517.

[15] Jarrett T. C., Ashton D. J., Fricker M., Johnson N. F. Interplay between function and structure in complex networks Phys. Rev. E 74 (2006) , 026116.

[16] Kirkpatrick D. G. and Radke J. D. A framework for computational morphology. In G. Toussaint, editor, Computational Geometry (1985) 217-248.

[17] Nakagaki T. Yamada H., Ueda T. Modulation of cellular rhythm and photoavoidance by oscillatory irradiation in the Physarum plasmodium. Biophysical Chemistry 82 (1999) 23-28.

[18] Nakagaki T., Yamada H., Ueda T. Interaction between cell shape and contraction pattern in the Physarum plasmodium, Biophysical Chemistry 84 (2000) 195-204.

[19] Nakagaki T., Smart behavior of true slime mold in a labyrinth. Research in Microbiology 152 (2001) 767-770.

[20] Nakagaki T., Yamada H., and Toth A., Path finding by tube morphogenesis in an amoeboid organism. Biophysical Chemistry 92 (2001) 47-52.

[21] Nakagaki T., Iima M., Ueda T., Nishiura y., Saigusa T., Tero A., Kobayashi R., Showalter K. Minimum-risk path finding by an adaptive amoeba network. Physical Review Letters 99 (2007) 068104.

[22] Nesetril J., Milkova E., Nesetrilova H., Otakar Boruvka on minimum spanning tree problem, Discrete Mathematics 233 (2001) 3-36.

[23] Matula D. W. and Sokal R. R. Properties of Gabriel graphs relevant to geographical variation research and the clustering of points in the same plane. Geographical Analysis 12 (1984) 205-222.

[24] Reyes D. R., Ghanem M. G., George M. Glow discharge in micro fluidic chips for visible analog computing. Lab on a Chip 1 (2002) 113-116.

[25] Schumann A. and Adamatzky A. Physarum spatial logic. In: Proc. 1th Int. Symp. on Symbolic and Numeric Algorithms for Scientific Computing (Timisoara, Romania, September 26-29, 2009).

[26] Shirakawa T., Adamatzky A., Gunji Y.-P., Miyake Y. On simultaneous construction of Voronoi diagram and Delaunay triangulation by Physarum polycephalum. Int. J. Bifurcation and Chaos (2009), in press.

[27] Stephenson S. L. and Stempen H. Myxomycetes: A Handbook of Slime Molds. (Timber Press, 2000).

[28] Toussaint G. T., The relative neighborhood graph of a finite planar set, Pattern Recognition 12 (1980) 261-268.

[29] Tsuda S., Aono M., Gunji Y.-P. Robust and emergent Physarum logicalcomputing. Biosystems 73 (2004) 45-55. 
[30] Watanabe D. A study on analyzing the road network pattern using proximity graphs. J of the City Planning Institute of Japan 40 (2005) 133-138.

[31] Watanabe D. Evaluating the configuration and the travel efficiency on proximity graphs as transportation networks. Forma 23 (2008) 81-87. 